\title{
DISFRAC Version 2.0 Users Guide
}

\section{$1 / 31 / 2013$}

\section{Prepared by}

K. B. Cochran ${ }^{1}$, M. Erickson ${ }^{2}$, P.T. Williams ${ }^{1}$, H. B. Klasky ${ }^{1}$ and B. R. Bass ${ }^{1}$

${ }^{1}$ Oak Ridge National Laboratory

Managed by UT-Battelle, LLC

Oak Ridge, TN 37831-8063

${ }^{2}$ Phoenix Engineering Associates, Inc

Davidsonville, MD 21035 


\section{DOCUMENT AVAILABILITY}

Reports produced after January 1, 1996, are generally available free via the U.S. Department of Energy (DOE) Information Bridge.

Web site http://www.osti.gov/bridge

Reports produced before January 1, 1996, may be purchased by members of the public from the following source.

National Technical Information Service
5285 Port Royal Road
Springfield, VA 22161
Telephone 703-605-6000 (1-800-553-6847)
TDD 703-487-4639
Fax 703-605-6900
E-mail info@ntis.gov
Web site http://www.ntis.gov/support/ordernowabout.htm

Reports are available to DOE employees, DOE contractors, Energy Technology Data Exchange (ETDE) representatives, and International Nuclear Information System (INIS) representatives from the following source.

Office of Scientific and Technical Information

P.O. Box 62

Oak Ridge, TN 37831

Telephone 865-576-8401

Fax 865-576-5728

E-mail reports@osti.gov

Web site http://www.osti.gov/contact.html

This report was prepared as an account of work sponsored by an agency of the United States Government. Neither the United States Government nor any agency thereof, nor any of their employees, makes any warranty, express or implied, or assumes any legal liability or responsibility for the accuracy, completeness, or usefulness of any information, apparatus, product, or process disclosed, or represents that its use would not infringe privately owned rights. Reference herein to any specific commercial product, process, or service by trade name, trademark, manufacturer, or otherwise, does not necessarily constitute or imply its endorsement, recommendation, or favoring by the United States Government or any agency thereof. The views and opinions of authors expressed herein do not necessarily state or reflect those of the United States Government or any agency thereof. 


\title{
DISFRAC VERSION 2.0 USERS GUIDE
}

\author{
Kristine Cochran \\ Marjorie Erickson \\ Paul Williams \\ Hilda Klasky \\ Richard Bass
}

Date Published: January 2013

\author{
Prepared by \\ OAK RIDGE NATIONAL LABORATORY \\ Oak Ridge, Tennessee 37831-6283 \\ managed by \\ UT-BATTELLE, LLC \\ for the \\ U.S. DEPARTMENT OF ENERGY \\ under contract DE-AC05-00OR22725
}

with support from Rolls-Royce Power Engineering Plc under contract no.PO 5200018124 



\section{CONTENTS}

Page

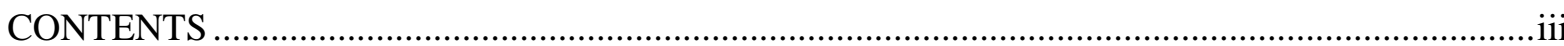

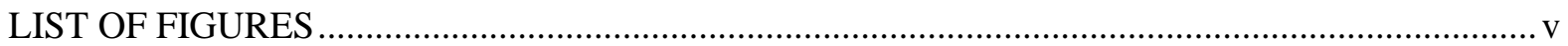

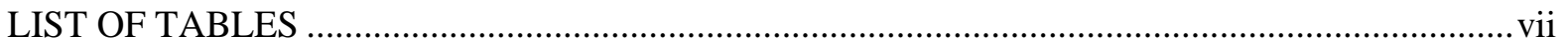

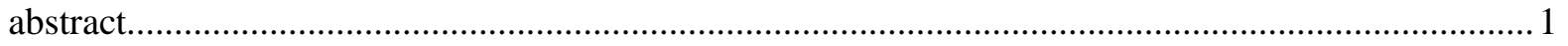

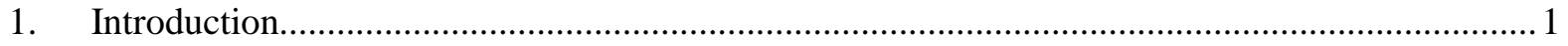

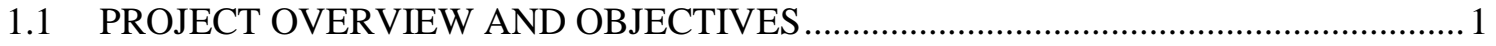

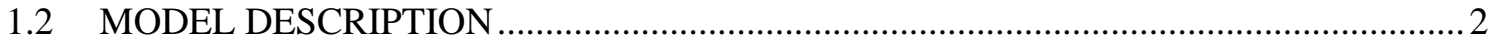

1.3 MODEL FOUNDATIONS, ASSUMPTIONS AND LIMITATIONS ….......................... 4

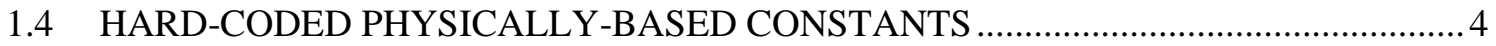

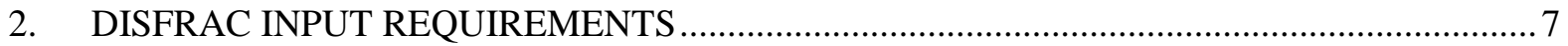

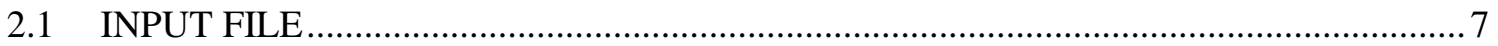

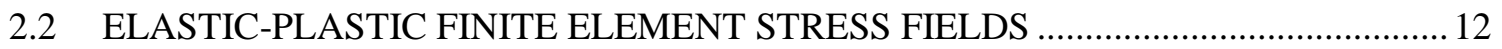

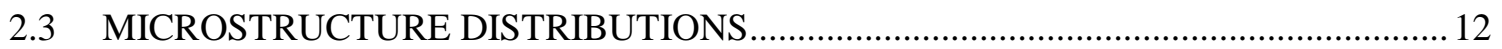

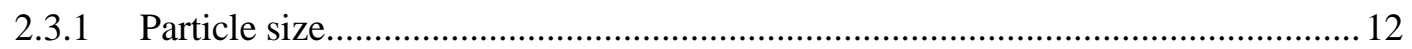

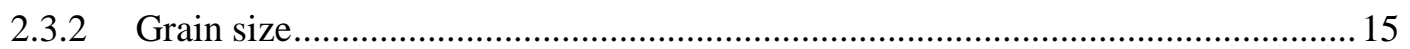

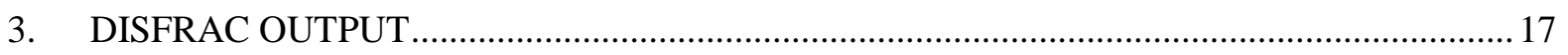

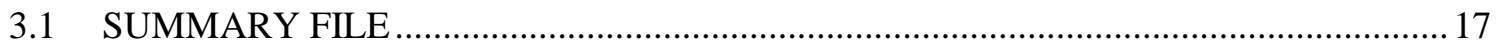

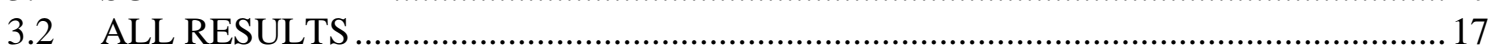

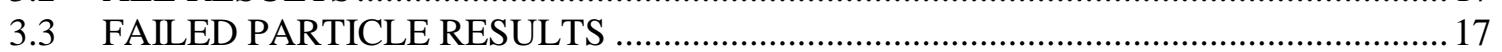

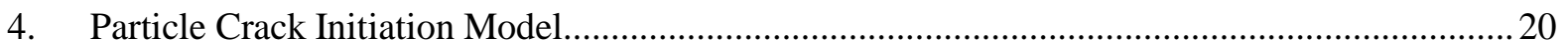

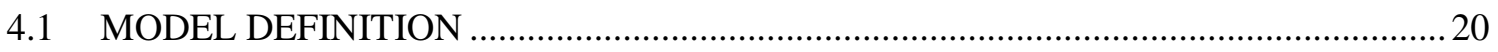

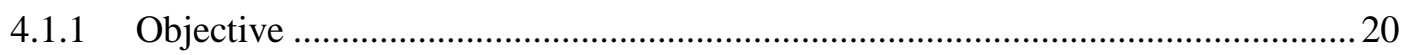

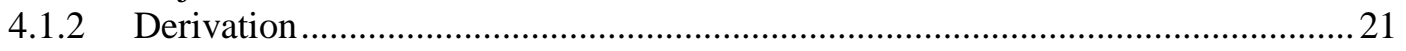

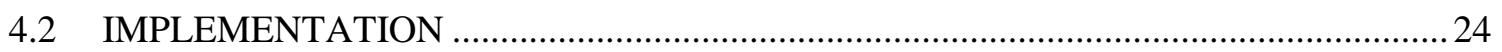

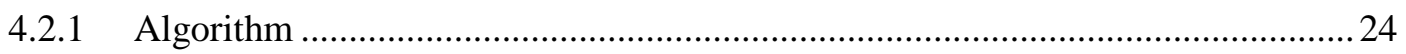

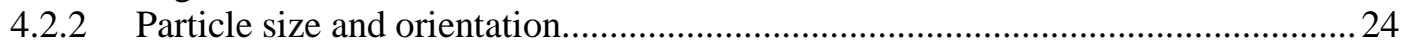

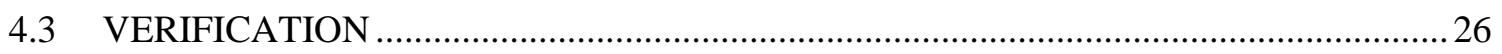

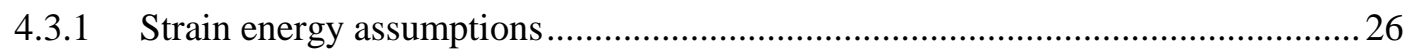

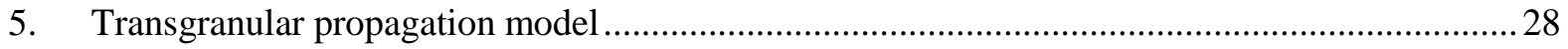

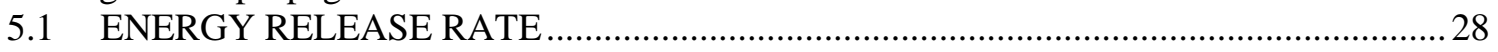

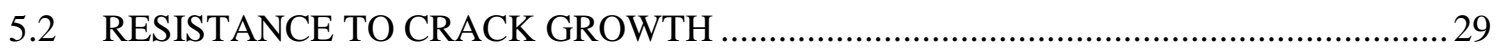

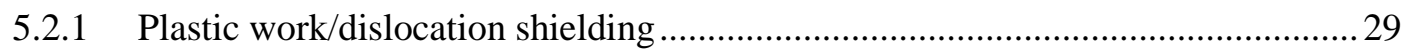

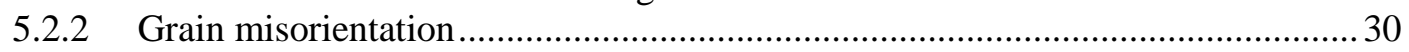

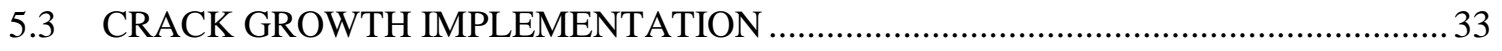

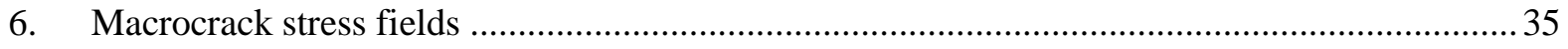

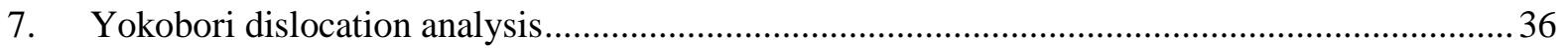

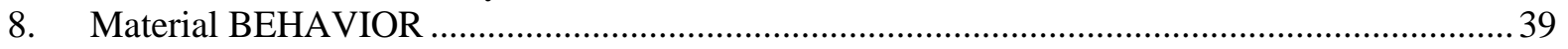

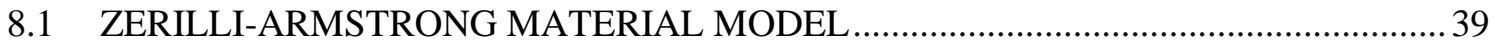

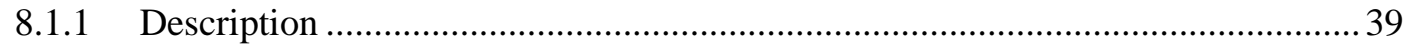

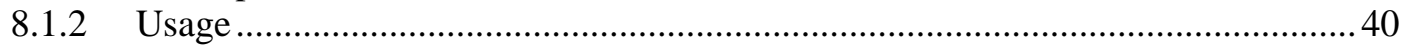

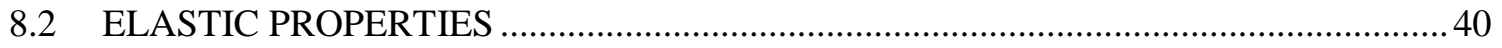

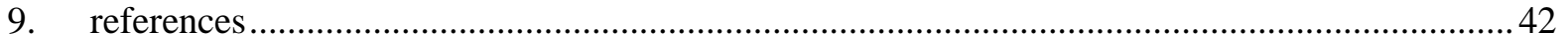

Appendix A. Linear elastic fracture mechanics (LEFM) background information.............................4 44 


\section{LIST OF FIGURES}

Figure

Figure 1. Macroscopic and microscopic geometry in DISFRAC model (not to scale)............3

Figure 2. DISFRAC V2.0 Flow Diagram..................................................................6

Figure 3. Particle size relationships for Rolls Royce Material A data set and

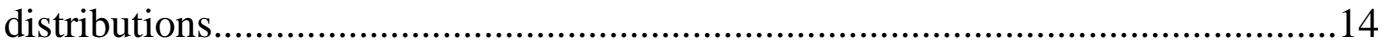

Figure 4. Cumulative distributions of various particle attributes. ......................................15

Figure 5. Cumulative and density distributions for grain diameter. ..................................16

Figure 6. Strain energy definition for Zerilli-Armstrong model ......................................22

Figure 7. Particle shape and orientation definitions............................................................25

Figure 8. Assessing the accuracy of the von Mises and plastic equivalent strain method for computing strain energy density. ...............................................................27

Figure 9. Angle between adjacent cleavage planes. .........................................................29

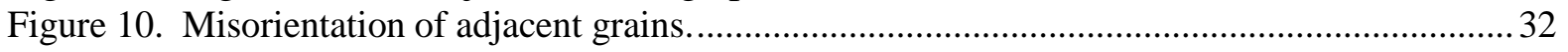

Figure 11. Grain boundary separation. Only a single breakthrough point shown............................... 32

Figure 12. Persistent grain boundary area for multiple breakthrough points .....................................33

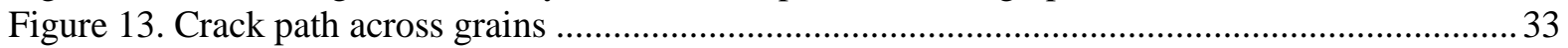

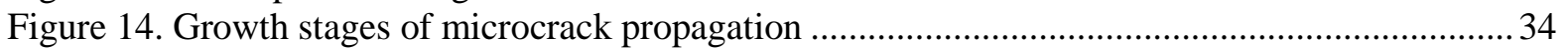

Figure 15. Modified boundary layer (MBL) finite element model (FEM) mesh .............................. 35

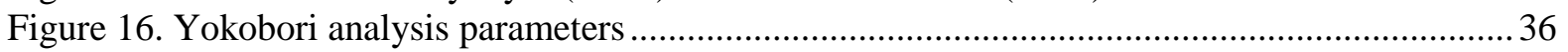

Figure 17. Angle definitions and coefficient dependence on $\alpha$ for stress-intensity factors of crack with infinitesimal direction change. 



\section{LIST OF TABLES}

Table

Page

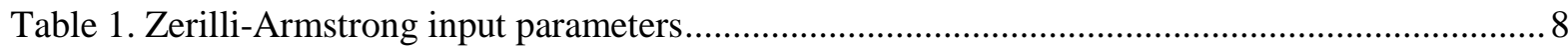

Table 2. Material model and constraint combinations currently available in DISFRAC .................... 12

Table 3. Rolls Royce Material A 2012 particle size parameters for lognormal bivariate distributions 13

Table 4. Grain size statistics and Weibull parameters for Rolls Royce Material A ............................ 16

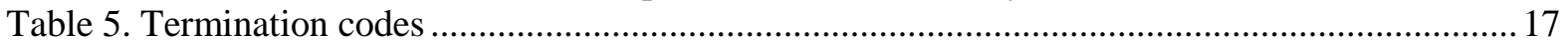

Table 6: Columns in all_results.out. Columns 21-30 are repeated for each grain boundary at which an

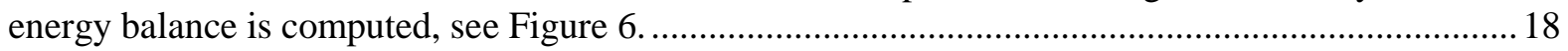





\begin{abstract}
DISFRAC is the implementation of a theoretical, multi-scale model for the prediction of fracture toughness in the ductile-to-brittle transition temperature (DBTT) region of ferritic steels. Empiricallyderived models of the DBTT region cannot legitimately be extrapolated beyond the range of existing fracture toughness data. DISFRAC requires only tensile properties and microstructural information as input, and thus allows for a wider range of application than empirical, toughness data dependent models. DISFRAC is also a framework for investigating the roles of various microstructural and macroscopic effects on fracture behavior, including carbide particle sizes, grain sizes, strain rates, and material condition.
\end{abstract}

DISFRAC's novel approach is to assess the interaction effects of macroscopic conditions (geometry, loading conditions) with variable microstructural features on cleavage crack initiation and propagation. The model addresses all stages of the fracture process, from microcrack initiation within a carbide particle, to propagation of that crack through grains and across grain boundaries, finally to catastrophic failure of the material. The DISFRAC procedure repeatedly performs a deterministic analysis of microcrack initiation and propagation within a macroscopic crack plastic zone to calculate a critical fracture toughness value for each microstructural geometry set.

The current version of DISFRAC, version 2.0, is a research code for developing and testing models related to cleavage fracture and transition toughness. The various models and computations have evolved significantly over the course of development and are expected to continue to evolve as testing and data collection continue. This document serves as a guide to the usage and theoretical foundations of DISFRAC v2.0. Feedback is welcomed and encouraged.

\title{
1. INTRODUCTION
}

\subsection{PROJECT OVERVIEW AND OBJECTIVES}

The importance of steel as a structural material has long motivated study of its fracture behavior. Of particular interest is the development of predictive models for nuclear reactor pressure vessel steel fracture safety under a variety of environmental conditions including radiation embrittlement. Current regulations require that U.S. power plants demonstrate a failure probability due to pressurized thermal shock of less than $5 \times 10^{-6}$ for the reactor pressure vessel ${ }^{1}$ through end-of-license. Approval of continued operation (i.e. re-licensing) depends on successful demonstration of fracture safety throughout the period of license extension.

Most of the currently available models of steel fracture behavior are empirically-derived, physicallyjustified models that depend on calibration factors to account for material variability, temperature and strain rate dependence, and geometry effects. The ASTM E1921 Master Curve 2 model requires testing to calibrate the model to characterize fracture toughness under a specific set of material and environmental conditions. The limited physical basis for most current models means they cannot be extrapolated beyond the empirical database used in their development. This predominantly empirical basis for regulations 
creates challenges for relicensing applications as regulation requires demonstration of fitness-for-service out to another 20 years of service and only very limited data exists for such highly irradiated steels.

Besides extending models beyond conditions covered by the database, a great deal of the current modeling effort is being focused on examining the distribution of toughness values of ferritic steels within the transition region, especially with regard to the behavior of the ASTM Master Curve ${ }^{2}$ at the lower bounds of the prescribed distribution3-4. Questions have been raised as to whether the Master Curve's theoretically-derived Weibull distribution provides an overly conservative estimation of the lower toughness values of the distribution. Comparisons between the Master Curve and data sets containing enough data to empirically define fracture toughness at very low failure probabilities appear to validate those claims of conservatism5. The underlying issue behind these questions deals with the nature of probabilistic fracture models in that such models aspire to generalize the full range of behavior of a class of materials using a numerical approximation to inherently limited data sets. Only after the data is approximated are attempts made to link the various statistical parameters and correlation factors to the physical phenomena of the material behavior at the microstructural level. As new data becomes available, the hope is that the original approximation is sufficiently adaptable to be able to account for the new data. It would be very useful in addressing the questions of Master Curve conservatism to have an accurate predictive model of the toughness distribution derived using the physical principles of deformation and fracture. Such a model would necessarily be deterministic in nature to predict the points within the distribution on a solid physical basis.

The model presented in the above paragraphs is the basis for the DISlocation-based FRACture (DISFRAC) [22] computer code being developed at the Oak Ridge National Laboratory (ORNL). The DISFRAC project's main objective is to develop a predictive model of toughness versus temperature based on the physical understanding of deformation and fracture. The model combines dislocation mechanics and continuum mechanics to capture the scatter of toughness values at each temperature. DISFRAC explicitly models the effects of both macroscopic variables (macrocrack size, specimen constraint, temperature, strain rate) and microstructure (size and orientation of specific grains, particle size, location, orientation). The computer code provides detailed computations and results for large numbers of potential microstructures. Thus, DISFRAC can evaluate the magnitude, scatter, and sensitivity of fracture toughness in ferritic steels.

\subsection{MODEL DESCRIPTION}

DISFRAC assesses the incidence of microcrack formation and subsequent brittle propagation, initiated at carbide particles within the plastic zone of a nearby macrocrack. There are two scales of physics involved: (1) the macroscopic, continuum stress-strain response to the macrocrack in specific loading and constraint conditions and (2) stress-strain conditions local to individual grains and based on dislocation motion. There are also two layers of computation: (1) deterministic analysis of microcrack initiation and propagation at a specified particle, through a given crack path and (2) Monte Carlo based analysis of random particle and grain sizes, shapes and locations selected from evidence-based distributions of these microstructural attributes.

The DISFRAC procedure considers each particle individually; performing a separate deterministic analysis for each. Figure 1 defines the pertinent geometric variables. The macroscopic specimen geometry (loading type, macrocrack size, T-stress) is pre-defined and constant across all deterministic analyses for a given simulation. Each deterministic analysis features a randomly selected particle (location, size, orientation) and crack path defined by subsequent grains (sizes, orientations).

Figure 2 illustrates the flow of the DISFRAC algorithm. For a given geometry set, DISFRAC determines the applied load required to fracture the particle. If excessive plasticity leads to void formation or violates 
small scale yielding, calculations cease for that particle. If conditions are met for microcrack initiation within the particle, the applied load remains constant. The microcrack then propagates in a step-wise fashion assuming growth momentarily halts at each successive grain boundary as the crack transitions to the orientation of the cleavage planes in the new grain.

A static equilibrium energy balance criterion assesses if the microcrack will cross a grain boundary. The criterion accounts for localized plasticity as well as tilt and twist misorientation between adjacent grains. If the microcrack propagation leads to specimen failure, DISFRAC records the $K_{c}$ associated with the applied load and moves on to a new geometry set. If the microcrack arrests before specimen failure, the algorithm proceeds to the next microgeometry case without recording a toughness value for the particle/grain set.

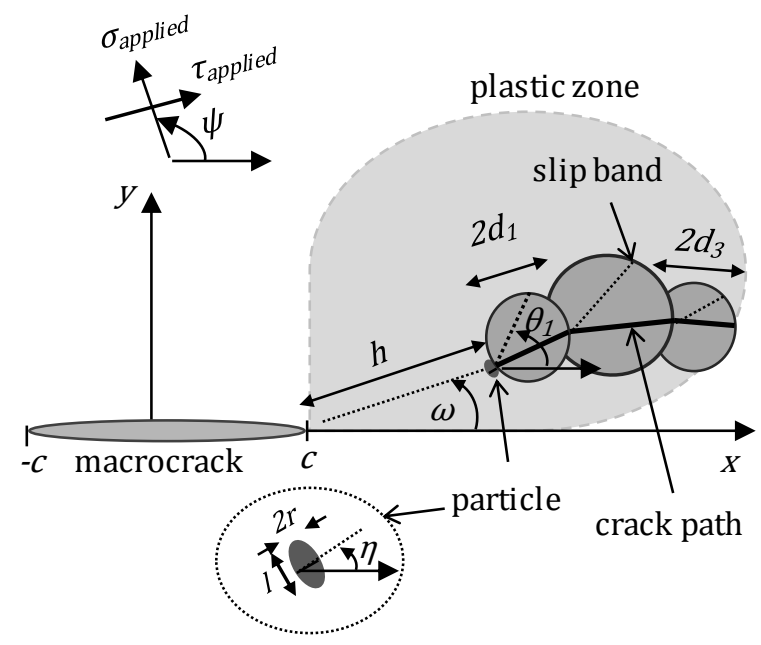

Figure 1. Macroscopic and microscopic geometry in DISFRAC model (not to scale).

The specific calculation steps within DISFRAC are shown in Figure 2, and described below:

A. Variable initialization. Sets the macrocrack size $(c)$, macrocrack tip radius $(\rho)$, and loading orientation $(\psi)$. These remain constant for the entire simulation.

B. Microgeometry set. Sets the particle size $(l)$, aspect ratio $(l / 2 r)$, location $(h, \omega)$, orientation $(\alpha, \eta)$ and the size $(d)$ and orientation $(\theta, \varphi)$ of the grains along the candidate crack path.

C. Small scale yielding limit. The maximum allowed applied load corresponds to a maximum stressintensity factor of 200MPa $\sqrt{\mathrm{m}}$, a conservative estimate based on Marston6.

D. Particle crack initiation. Section 4 describes in detail the particle crack initiation model (phase 0). DISFRAC computes the magnitude of the applied load such that the strain energy available to crack the particle equals the resistance to crack formation. The resistance includes surface energy and dissipative effects of dislocation motion in the adjacent grain.

E. Void formation. DISFRAC compares the equivalent plastic strain in the matrix at the failure load to a void nucleation strain. If the equivalent plastic strain is larger than the void nucleation strain, 
DISFRAC assumes a void would form before particle fracture occurs. The void nucleation strain is based the value used by Xia and Chen7.

F. Transboundary propagation. Section 5 describes in detail the transboundary propagation model. At the particle/grain boundary (phase 1) and each subsequent grain/grain boundary (phase 3), DISFRAC performs an energy balance analysis to determine if the microcrack will arrest at the boundary. DISFRAC assumes the microcrack stalls temporarily at each boundary; the model employs static equilibrium conditions.

G. Specimen failure. The microcrack propagation for the particle/grain set causes global specimen failure under two conditions. If the microcrack extends beyond the plastic zone caused by the initiation load, the specimen fails. For small-grained materials with low yield points, this condition becomes computationally onerous however. The user may define a maximum number of grains the crack must cross before specimen failure is inevitable. Sensitivity studies can guide the user in setting this parameter. Initial studies with a fine-grained material ( $4 \mu \mathrm{m}$ average grain diameter) indicate that if the microcrack extends through 8-10 grains, it will not arrest at any subsequent grain boundaries.

\subsection{MODEL FOUNDATIONS, ASSUMPTIONS AND LIMITATIONS}

- The microsystems (particle and grains) exist within a continuum-based deformation field of a macrocrack within a specimen.

- The continuum stress-strain fields act as the loading to inform the micromechanical (dislocationbased) calculations of the microsystem.

- Finite element analyses with a small-scale yielding boundary layer model provide the stress-strain fields for discrete sets of (a) material parameters, (b) strain-rate, (c) temperature and (d) constraint (T-stress).

- All analyses assume plane strain conditions.

- The microsystem creates localized linear-elastic stress-strain concentrations characterized by dislocation distributions.

- Calculation of the dislocation distributions assumes static equilibrium with the macroscopic stress state.

- The macrocrack is unaffected by the microsystem (there is no feedback from the microsystem to the continuum stress field calculation).

- The growing microcrack is represented by an equivalent straight microcrack between the defined endpoints, not the tortuous path created through the misaligned grains.

- The material response is characterized by the Zerilli-Armstrong material model ${ }^{17}$.

- Currently, only mode I loading (on the macrocrack) is treated.

\subsection{HARD-CODED PHYSICALLY-BASED CONSTANTS}

The following list provides the current assumed values of the various physical constants employed within DISFRAC. The constants are defined in more detail within the chapters corresponding to their specific model. All of these parameters have physical significance to the process of cleavage fracture as modeled in DISFRAC (i.e, they are not simply empirical constants). Nevertheless, uncertainty exists regarding the exact values or range of values. The next version of DISFRAC will treat these parameters as user input to facilitate sensitivity studies.

- $\gamma_{c}$ : carbide surface energy $=2.0 \mathrm{~J} / \mathrm{m}^{2}$ 
- $\gamma_{s}$ : ferrite surface energy of cleavage plane $=2.0 \mathrm{~J} / \mathrm{m}^{2}$

- $\gamma_{s, G B}$ : ferrite surface energy of grain boundary $=2.0 \mathrm{~J} / \mathrm{m}^{2}$

- $\delta_{G B}$ : critical separation of grain boundary $=1.8 \mathrm{E}-8 \mathrm{~m}$

- $n$, number of breakthrough points $=1$

- $\varepsilon_{\text {void }}$ : plastic strain for void nucleation $=0.04$

- $K_{\max }$ : ssy limit $=200 \mathrm{MPa} \mathrm{m}^{0.5}$

- $\beta$ : angle between slip and cleavage planes for $\mathrm{BCC}$ metal $=\pi / 4$ 


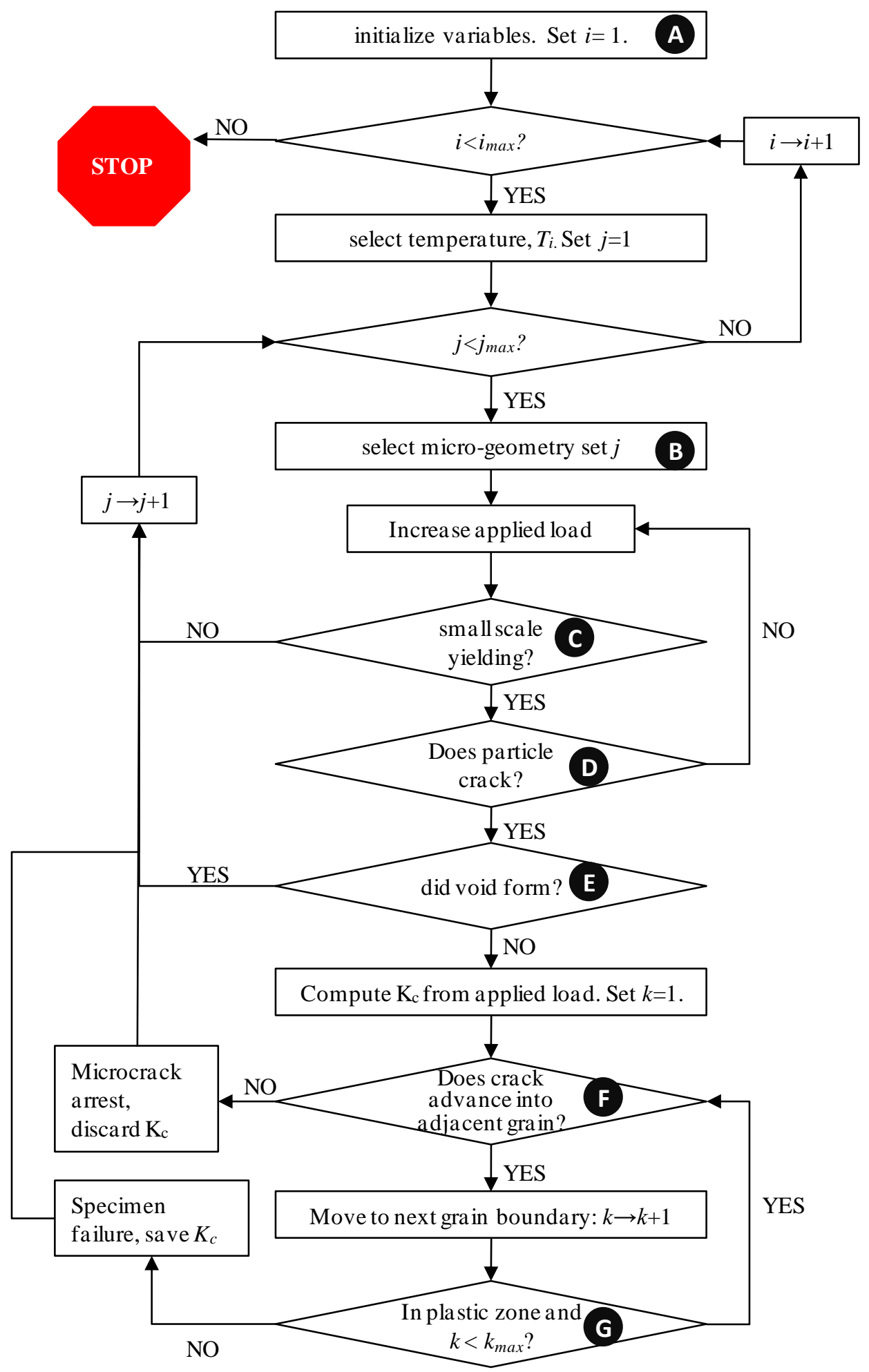

Figure 2. DISFRAC V2.0 Flow Diagram. The circled letters refer to specific calculations described above. 


\section{DISFRAC INPUT REQUIREMENTS}

Executing DISFRAC requires a single, text-based input file. Section 2.1 describes the necessary input values and structure of the file. This file is the only user-level input requirement. DISFRAC currently also employs indirect (hard-coded) input in the form of stress fields and microstructure distributions. Section 2.2 discusses the included stress fields, from associated finite element analyses. Section 2.3 presents the microstructural distributions employed in DISFRAC.

\subsection{INPUT FILE}

The foundation of DISFRAC user-input is a single structured text file. The input translator requires the input data in specific sequence and format and thus it is advisable to modify a sample input file rather than create one from scratch. A sample input file is included with the code distribution. The input file is presented and described line-by-line below.

Lines that begin with // are comments and not read by the input translator. All other lines must be in the proper order. Note that DISFRAC doesn't yet provide error checking on user-input.

\section{Input line 1: select output files and random number generator seed type}

There are 3 possible output files; the user selects which ones are actually written for the current analysis. The three files are described in section 2. The user may also toggle the case-wise screen output on and off. If screen output is on, data for each case is written to the screen and the simulation won't fully terminate (close the screen) until the user interacts with the screen at the end of the analysis. This allows the user to review the screen output, if desired, after a diagnostic analysis (low number of cases).

The random number generator seed can be set in two different ways. It can be a hard-coded constant that allows for simulation repeatability by always producing the same random variables for each case, for the same input file. Alternatively, the seed can be set randomly based on the date and clock time. This allows for simulation extension without repeating the same cases. For example, the user may run 10000 cases but decide later than 20000 cases are required. Using the random seed, the user may simply run another 10000 cases which will be distinct from the first 10000 cases, provided the desired total cases.

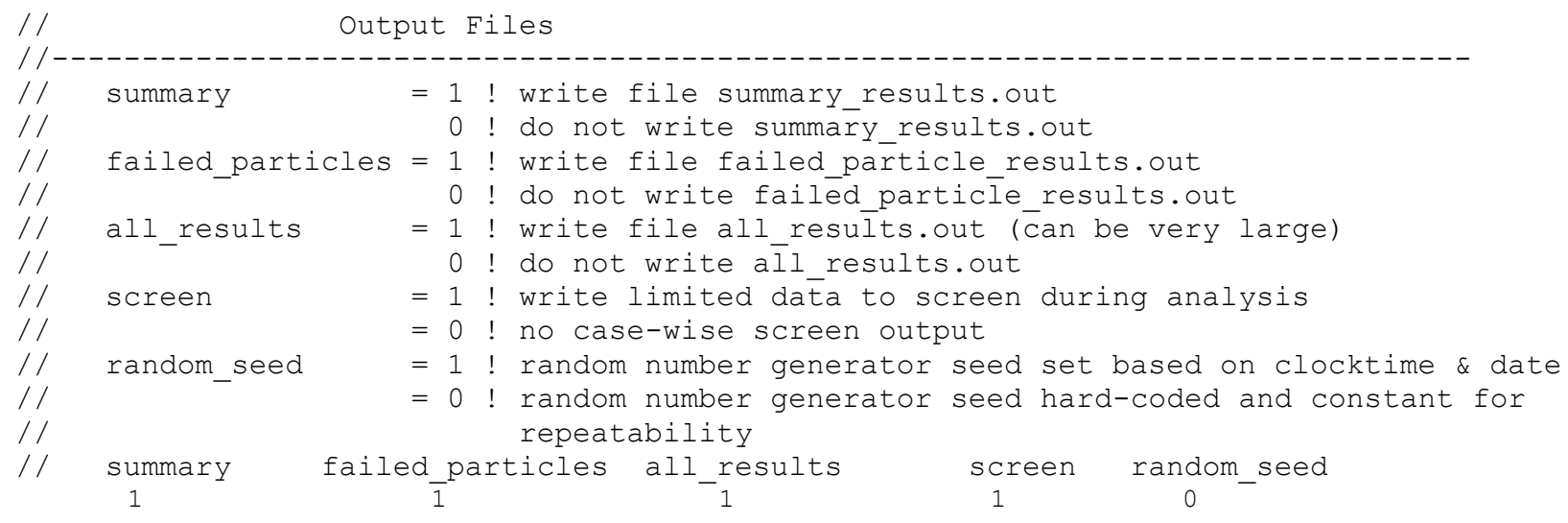




\section{DRAFT}

\section{Input line 2: constant input data}

For most applications, the only item in line 2 a user needs to modify is nsim; set it to the number of Monte Carlo trials requested for each temperature. The other items in line 2 allow the user to control the applied load, request deterministic (not random) geometry, or prevent propagation of the crack (activate only the initiation model).

If ifind $=0$, DISFRAC determines if the crack initiates (and propagates) at the fixed applied load given by sigma_app. Otherwise sigma_app and tau_app are not used; DISFRAC determines the applied load needed to initiate the microcrack via the initiation model (NB: code does not currently handle or apply non-zero tau_app).

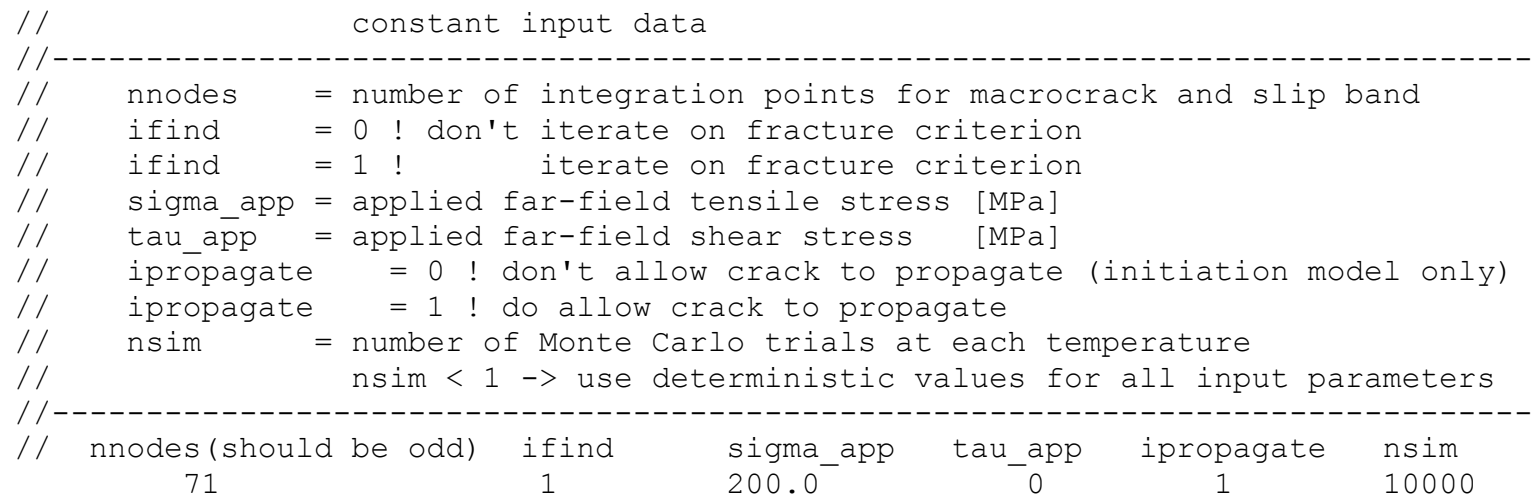

\section{Input line 3: parameters for Zerilli-Armstrong constitutive model}

The first 8 parameters specify the Zerilli-Armstrong constants for the user's selected material, according to the following equation.

$$
\sigma_{Z A}=\sigma_{G}+k_{y} / \sqrt{2 d}+B_{0} e^{-\left(\alpha_{0}-\alpha_{1} \ln \dot{\epsilon}\right) T} \sqrt{\epsilon}+B e^{-\left(\beta_{0}-\beta_{1} \ln \dot{\epsilon}\right) T}
$$

Table 1 links the parameters in the equation with their input names and provides the values for 2 specific materials. In the above equation, $2 d$ is the average grain diameter (input later), $\epsilon$ is the current equivalent strain, $\dot{\epsilon}$ is the (user-defined) strain rate, $T$ is the temperature (in Kelvin).

Table 1. Zerilli-Armstrong input parameters

\begin{tabular}{|l|l|l|l|}
\hline Parameter & Input name & Eurosteel & Shoreham plate \\
\hline$\sigma_{G}[\mathrm{MPa}]$ & sigG & 0.0 & 145 \\
\hline$k_{y}[\mathrm{MPa} \sqrt{\mathrm{m}}]$ & $\mathrm{k} \_\mathrm{y}$ & 1.205 & 0.7 \\
\hline$B_{0}[\mathrm{MPa}]$ & B0 & 881.2 & 229 \\
\hline$\alpha_{0}[1 / \mathrm{K}]$ & alpha0 & 0.0 & $-3.43 \mathrm{e}-03$ \\
\hline$\alpha_{1}[1 / \mathrm{K}]$ & alpha1 & 0.0 & $-4.83 \mathrm{e}-05$ \\
\hline$B[\mathrm{MPa}]$ & B & 1044.7 & 1428 \\
\hline$\beta_{0}[1 / \mathrm{K}]$ & beta0 & $9.03 \mathrm{e}-03$ & $6.43 \mathrm{e}-03$ \\
\hline$\beta_{1}[1 / \mathrm{K}]$ & beta1 & $3.36 \mathrm{e}-04$ & $2.15 \mathrm{e}-04$ \\
\hline
\end{tabular}




\section{DRAFT}

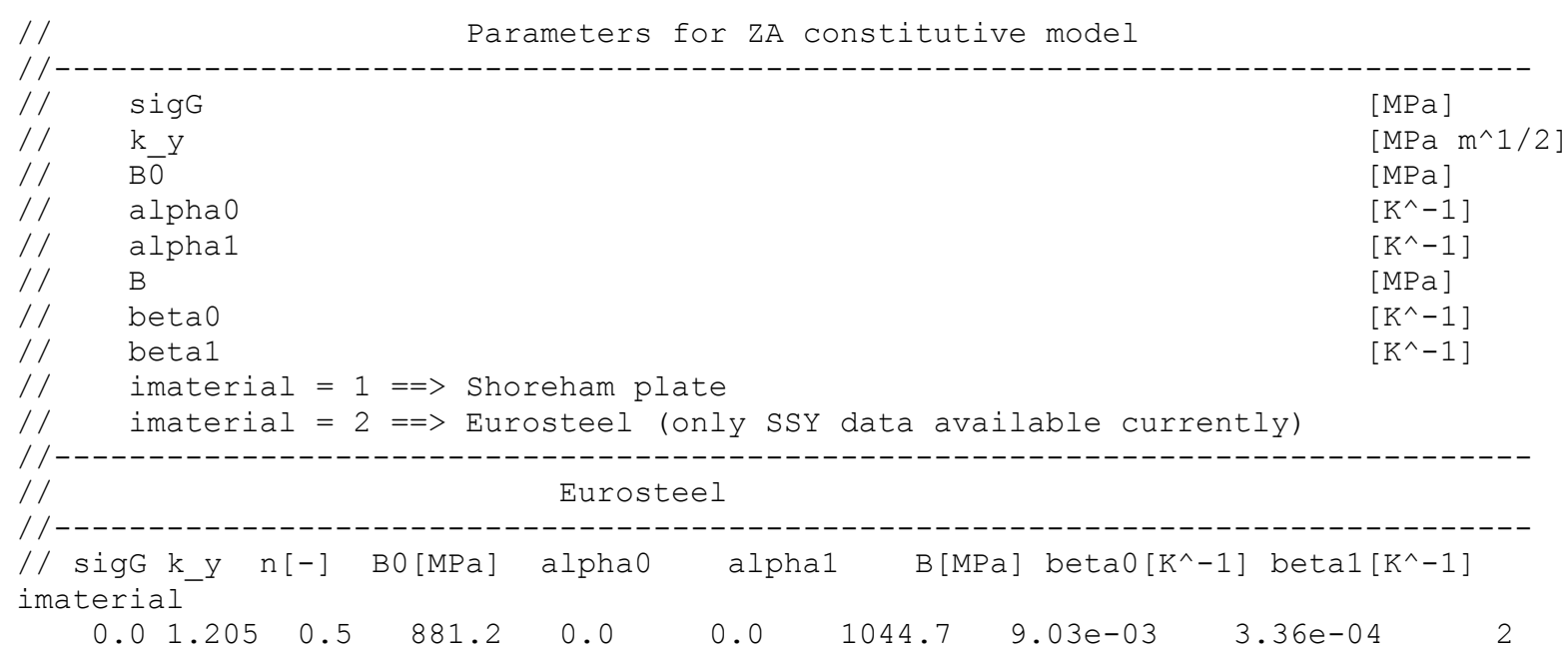

\section{Input line 4: macroscopic geometry inputs}

The macrocrack and applied load orientation and specimen constraint require the 4 parameters as listed below. Specimen-specific constraint is applied via in the MBL finite element model by the biaxiality ratio, $\beta=T \sqrt{\pi c} / K_{I}$, where $T$ is the T-stress and $K_{I}$ is the applied mode I stress-intensity. Only a limited set of material and constraint options is currently available, see section 2.2.

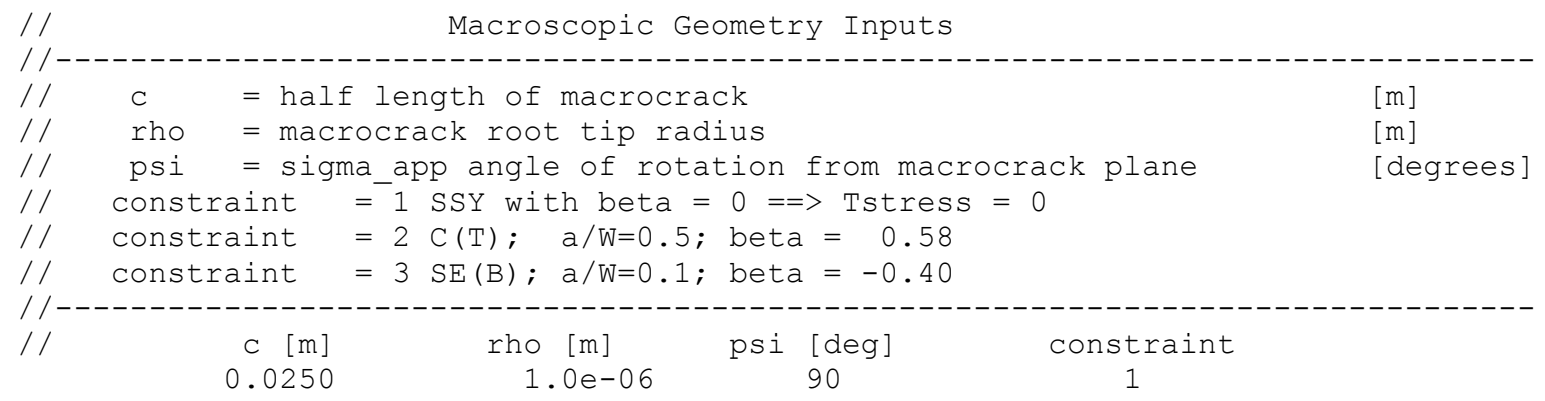

\section{Input line 5: material inputs}

The parameters on this line specify additional material properties and the strain rate. Note that edot_macro is the strain rate of the background material. DISFRAC uses edot_macro directly in all Zerilli-Armstrong calculations and indirectly via the finite element calculations for the elastic-plastic stress field. The microscopic strain rate, edot_micro, is not currently used.

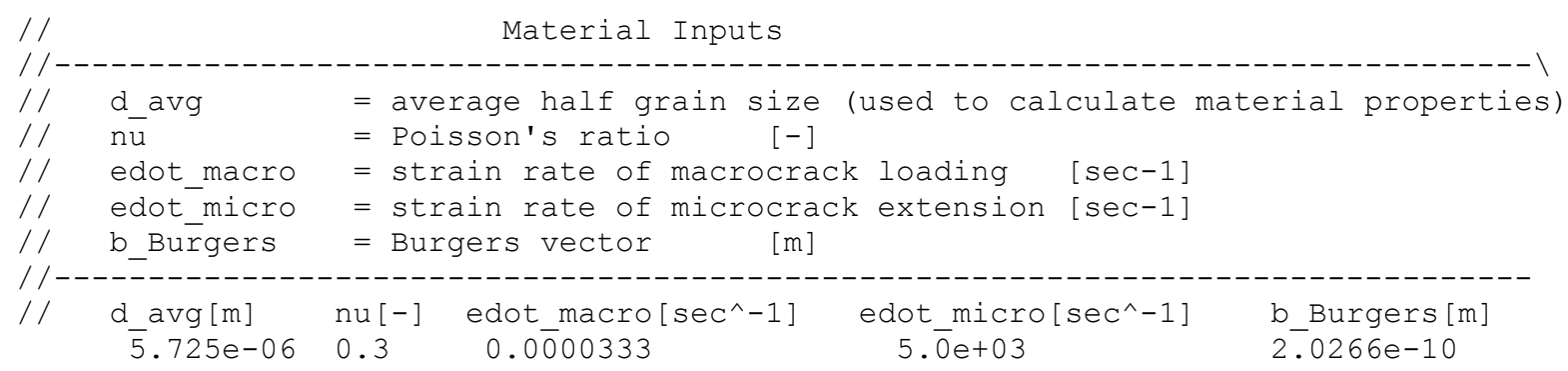




\section{Input line 6: temperature range}

Define the range of temperatures by a maximum temperature, minimum temperature and either an increment or the number of equally spaced temperature values. The currently available range of temperatures is $-150^{\circ} \mathrm{C}$ to $+25^{\circ} \mathrm{C}$. Note that if temperature increment is selected (iflag $=1$ ) and the input increment does not evenly divide the desired range, then the maximum temperature will not be userselected maximum. There is currently no error-checking on the bounds or intervals.

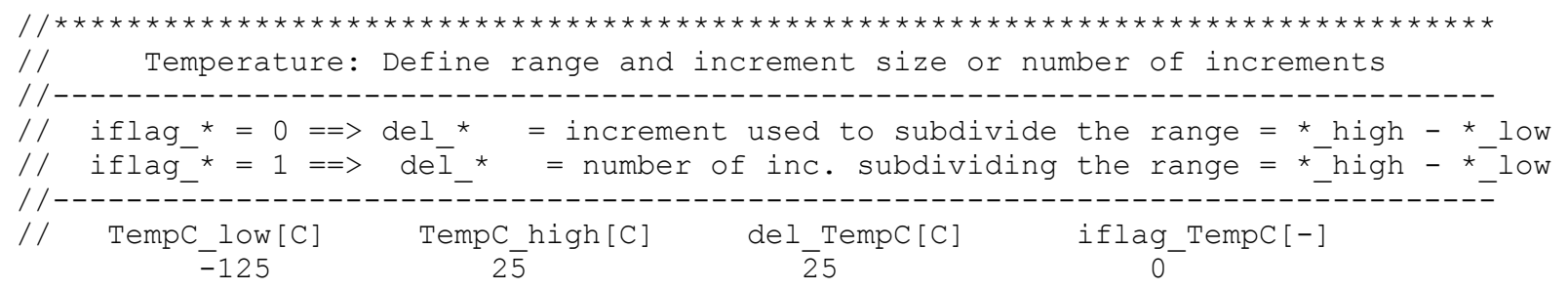

\section{Input line 7: deterministic particle geometry}

Specify the particle geometry, position and orientation for a deterministic simulation $(\mathrm{nsim}<1)$. The input translator ignores this line for Monte Carlo simulations. The angular position of the particle $(\omega)$ must be between -90 and $90^{\circ}$. Note that $l$ should be greater than or equal to $t$ to conform to the notation and assumptions in the particle initiation model, although it will not cause numerical or theoretical problems if $t>l$.

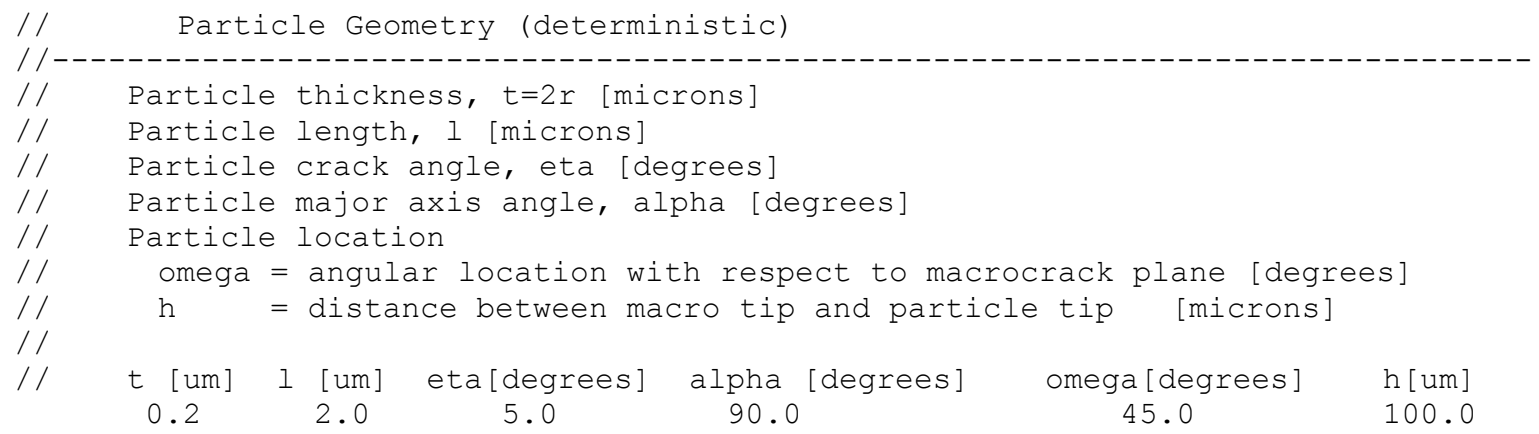

\section{Input line 8: Monte Carlo (random) particle geometry}

Specify the particle geometry distributions and range of positions and orientations. Currently there is only one particle size distribution included. There is also only one option for particle orientation (NOT correlated with grain structure). In the future, this parameter will allow for cases in which the particle major axis orientation is not completely random (to account for Kurdjumov-Sachs relationships).

The boundary sets the limits for the position of particle. For bound_shape 1, specify the boundary in polar coordinates, creating an arc-shaped region populated with particles. The arc may include any of the angular region within -90 to $+90^{\circ}$. For a rectangular region (bound_shape $=2$ ), only positive values are considered. The minimum $x$ and $y$ or minimum $h$ define an exclusion zone, to prevent consideration of particles too close to the macrocrack tip. 


\section{DRAFT}

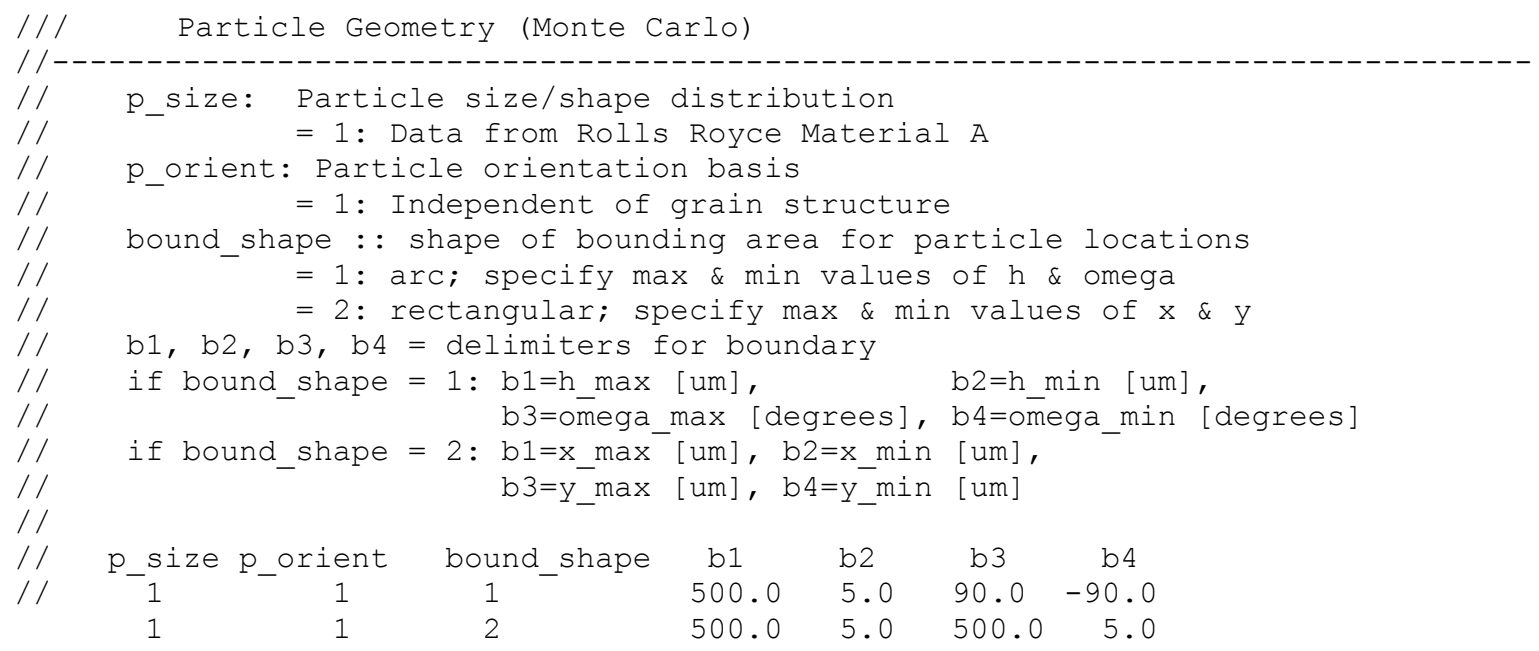

\section{Input line 9: maximum number of grains and grain distribution}

The parameter max_grains sets the maximum number of grains to propagate the crack across for each deterministic simulation. For a Monte Carlo simulation, set this to a reasonable number ( 10); grain sizes and orientations will be determined randomly from the corresponding distributions. To have a userdefined grain path, set this to the desired number of grains and then set the path starting with input line 10 (see below). The parameter $g_{-}$size sets the specific distribution for grain sizes.

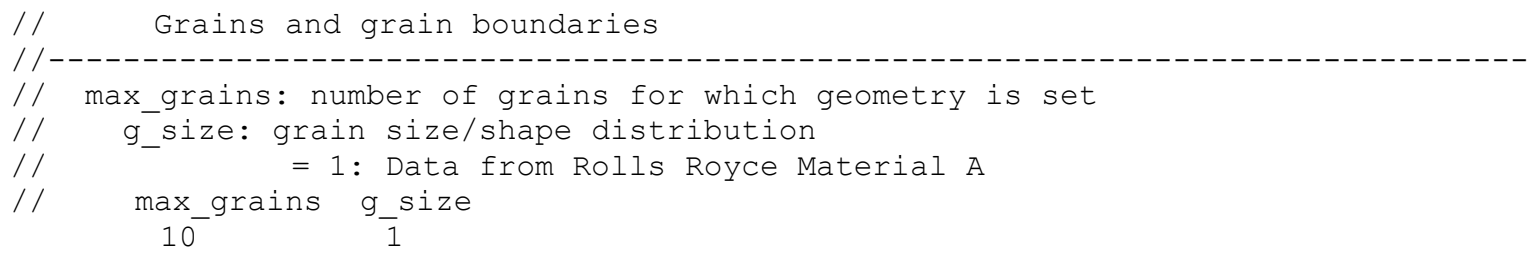

\section{Input lines 10 and on: grain geometries}

Describe the grain sizes and orientations for fully deterministic (user-specified) grain path. All input is ignored in a Monte Carlo analysis. Note that grain size and orientation are specified for each grain, while the twist angle is relative (the out-of-plane angular change between cleavage planes). Thus each input line $i$ provides the size of grain $i$, the orientation of grain $i$, and the twist angle between grain $i-1$ and grain $i$.

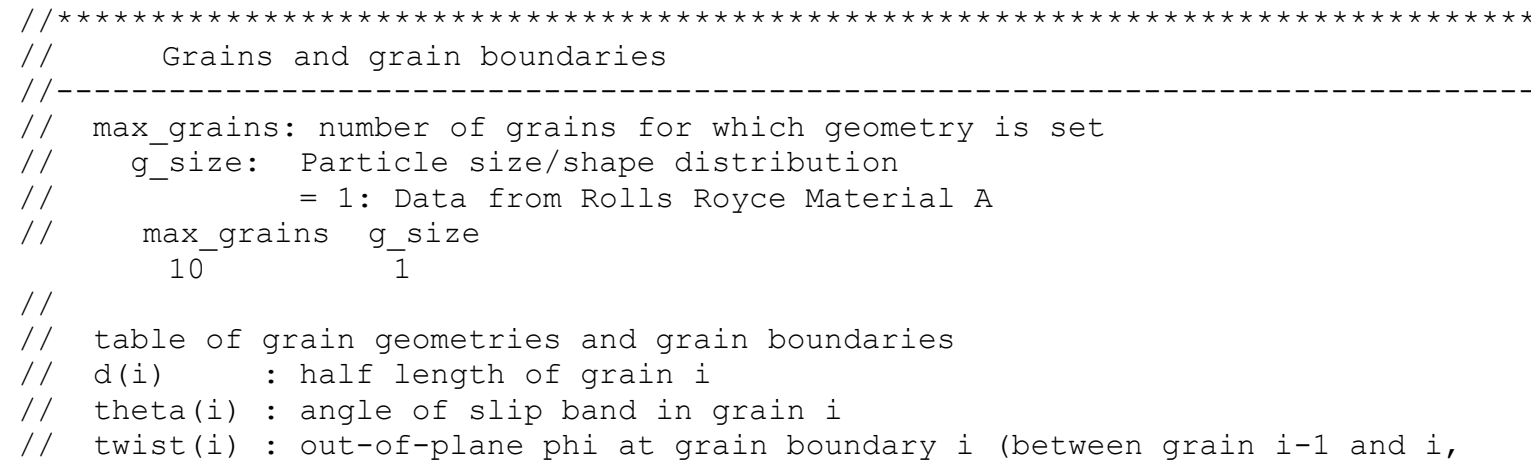




\section{DRAFT}

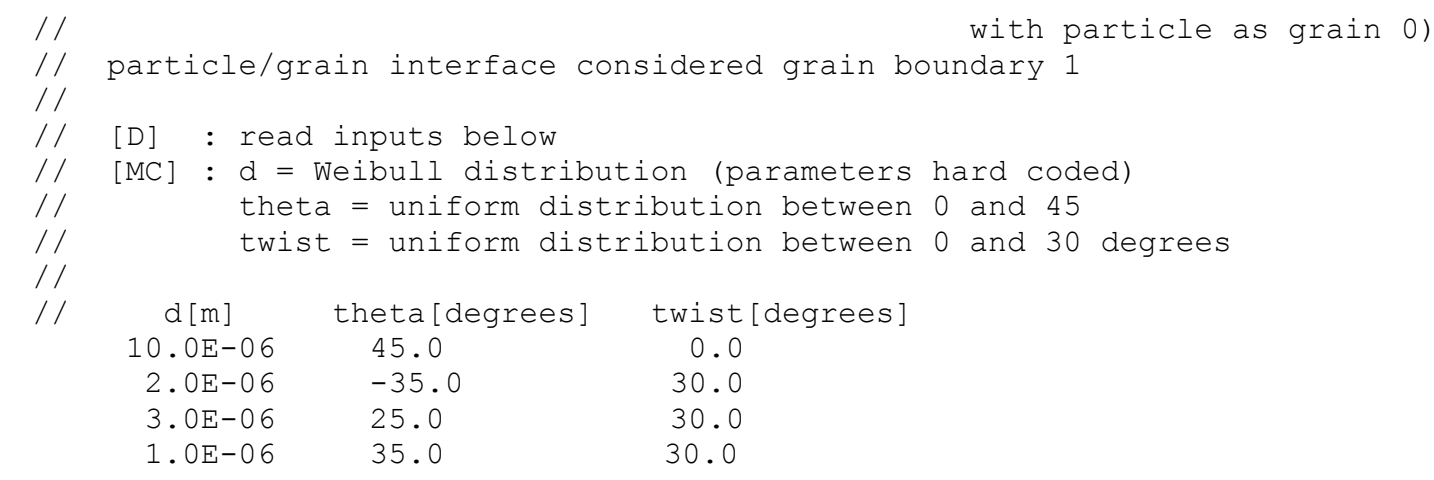

\subsection{ELASTIC-PLASTIC FINITE ELEMENT STRESS FIELDS}

The elastic-plastic finite element stress fields are not user-level input. Nevertheless, it is important to consider them to be input in some sense since they constrain some of the user input described above. For a given load, position relative to the macrocrack tip, and temperature, DISFRAC employs a combination table look-up/interpolation strategy to compute the stress field components. The look-up values come from series of finite element calculations that are specific for a given material (Zerilli-Armstrong parameter set), strain rate, and constraint (T-stress). The currently available data sets include the constraint and material combinations in the table below; all analyses employed the same strain rate, $\dot{\varepsilon}=3.33 \mathrm{E}-05 \mathrm{sec}^{-1}$. More information on the generation of the macroscopic stress and strain fields in DISFRAC can be found in section 6.

Table 2. Material model and constraint combinations currently available in DISFRAC

\begin{tabular}{|l|c|c|c|}
\hline Material model & $\begin{array}{c}\text { Small scale yielding (SSY) } \\
\boldsymbol{\beta}=\mathbf{0}\end{array}$ & $\begin{array}{c}\text { High constraint compact (CT) } \\
\mathbf{a} / \mathbf{W}=\mathbf{0 . 5}, \boldsymbol{\beta}=\mathbf{0 . 5 8}\end{array}$ & $\begin{array}{c}\text { Shallow bend bar (BB) } \\
\mathbf{a} / \mathbf{W}=\mathbf{0 . 1}, \boldsymbol{\beta}=\mathbf{- 0 . 4}\end{array}$ \\
\hline Eurosteel & $\checkmark$ & not available & not available \\
\hline Shoreham Plate & $\checkmark$ & $\checkmark$ & $\checkmark$ \\
\hline
\end{tabular}

\subsection{MICROSTRUCTURE DISTRIBUTIONS}

In Monte Carlo mode, DISFRAC selects particle and grain sizes randomly from known distributions. The specific distributions are selected via the $p \_$size and $g \_$size parameters.

\subsubsection{Particle size}

As described in section 4, the particle crack initiation model requires the length and aspect ratio (or length and width) for each particle. In the input file, the user may select one of the following particle size distribution models.

\subsubsection{Rolls Royce Material A $2012\left(p \_s i z e=1\right)$}

The data from Rolls Royce for this material provided particle length, width, aspect ratio, and area information for 5628 carbide particles. DISFRAC employs lognormal bivariate distributions fit to the length and aspect ratio data to characterize the particles. Independent lognormal distributions were fit to the major axis $(l)$ and aspect ratio $\left(a_{r}\right)$ data. First, the data are shifted so that the minimum values are zero. For example, for each major axis length measurement $i, l_{\text {shift }}(i)=l(i)-\delta_{l}$, where $\delta_{l}=\min (l)$. The logarithmic mean $\left(\mu_{\text {log }}\right)$ and standard deviation $\left(\sigma_{\text {log }}\right)$ were calculated from the arithmetic mean $(a v g)$ and standard deviation (stdev) of the shifted data. 


$$
\begin{gathered}
\mu_{\log }=\ln (a v g)-0.5 \ln \left[1+\left(\frac{\text { stdev }}{a v g}\right)^{2}\right] \\
\sigma_{\log }=\sqrt{\ln \left[1+\left(\frac{s t d e v}{a v g}\right)^{2}\right]}
\end{gathered}
$$

Table 3 lists the relevant parameters for the two data sets. The correlation coefficient for these sets of data is $\rho_{\text {cor }}=0.530322$. The logarithmic correlation constant is $\rho_{\text {logcor }}=0.619525$, as computed via the following equation.

$$
\rho_{\text {logcor }}=\frac{\ln \left\{\rho_{\text {cor }} \sqrt{\left[\exp \left(\sigma_{\log (l)}^{2}+\sigma_{\log \left(a_{r}\right)}^{2}\right)-\exp \left(\sigma_{\log (l)}^{2}\right)-\exp \left(\sigma_{\log \left(a_{r}\right)}^{2}\right)+1\right]+1}\right\}}{\sigma_{\log (l)} \sigma_{\log \left(a_{r}\right)}}
$$

Table 3. Rolls Royce Material A 2012 particle size parameters for lognormal bivariate distributions

\begin{tabular}{|l|l|l|l|l|c|}
\hline & \multicolumn{1}{|c|}{ shift, $\boldsymbol{\delta}(\boldsymbol{\mu m})$} & \multicolumn{1}{|c|}{ avg $(\boldsymbol{\mu m})$} & \multicolumn{1}{c|}{ stdev $(\boldsymbol{\mu m})$} & $\boldsymbol{\mu}_{\log }$ & $\boldsymbol{\sigma}_{\log }$ \\
\hline major axis $(\boldsymbol{l})$ & 0.07018 & 0.145884 & 0.161355 & -2.32445 & 0.893876 \\
\hline aspect ratio $\left(\boldsymbol{a}_{\boldsymbol{r}}\right)$ & 1 & 1.929403 & 1.901889 & 0.317767 & 0.823946 \\
\hline
\end{tabular}

The bivariate sampling occurs as follows. Two random samples from a standard normal distribution (i.e., mean $=0$, standard deviation $=1$ ) are selected; $N_{l}$ and $N_{2}$. Then, the corresponding length and aspect ratio are computed using the following equations.

$$
\begin{gathered}
Y_{(l)}=\mu_{\log (l)}+N_{1} \sigma_{\log (l)} \\
Y_{\left(a_{r}\right)}=\mu_{\log \left(a_{r}\right)}+\left(N_{1} \sigma_{\log \left(a_{r}\right)} \rho_{\text {logcor }}\right)+\left(N_{2} \sigma_{\log \left(a_{r}\right)} \sqrt{1-\rho_{\log c o r}^{2}}\right) \\
X_{(l)}=\exp \left[Y_{(l)}\right], \quad X_{\left(a_{r}\right)}=\exp \left[Y_{\left(a_{r}\right)}\right]
\end{gathered}
$$

$X_{(l)}$ and $X_{\left(a_{r}\right)}$ are the values of $l$ and $a_{r}$, respectively, corresponding to the two random samples $N_{l}$ and $N_{2}$, and the calibrated parameters. The particle thickness, $t$, is $l / a_{r}$. DISFRAC caps the distributions such that $l<l_{\max }=2.2 \mu \mathrm{m}$ and $t<t_{\max }=0.66 \mu \mathrm{m}$; the maximum sizes found in the Rolls Royce data for this material. 
Figure 3 shows scatter plots relating the thickness and aspect ratio to the particle length, for both the data and the derived distribution in DISFRAC. Figure 4 presents the cumulative particle distribution as a function of particle length, thickness, aspect ratio and area. The particle area based on the implemented distributions corresponds to an ellipse with major and minor axis lengths set by the particle length and thickness.

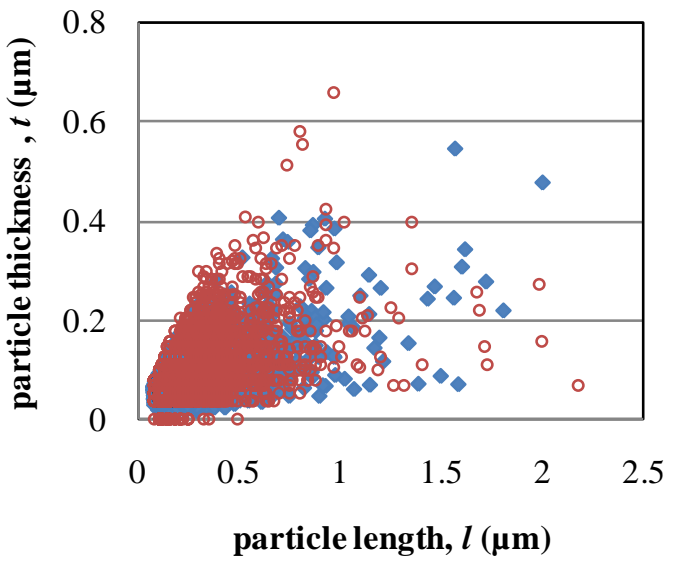

- DISFRAC bivariate lognormal distribution

- Rolls Royce Material A data

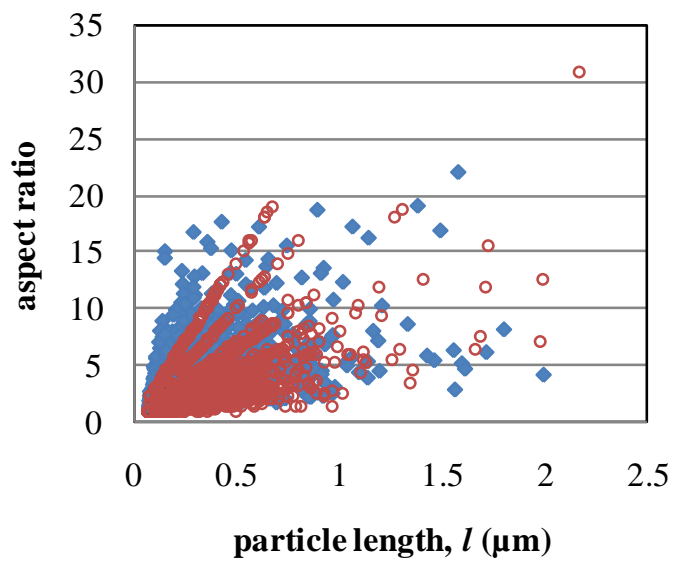

- DISFRAC bivariate lognormal distribution

- Rolls Royce MaterialA data

Figure 3. Particle size relationships for Rolls Royce Material A data set and distributions. 

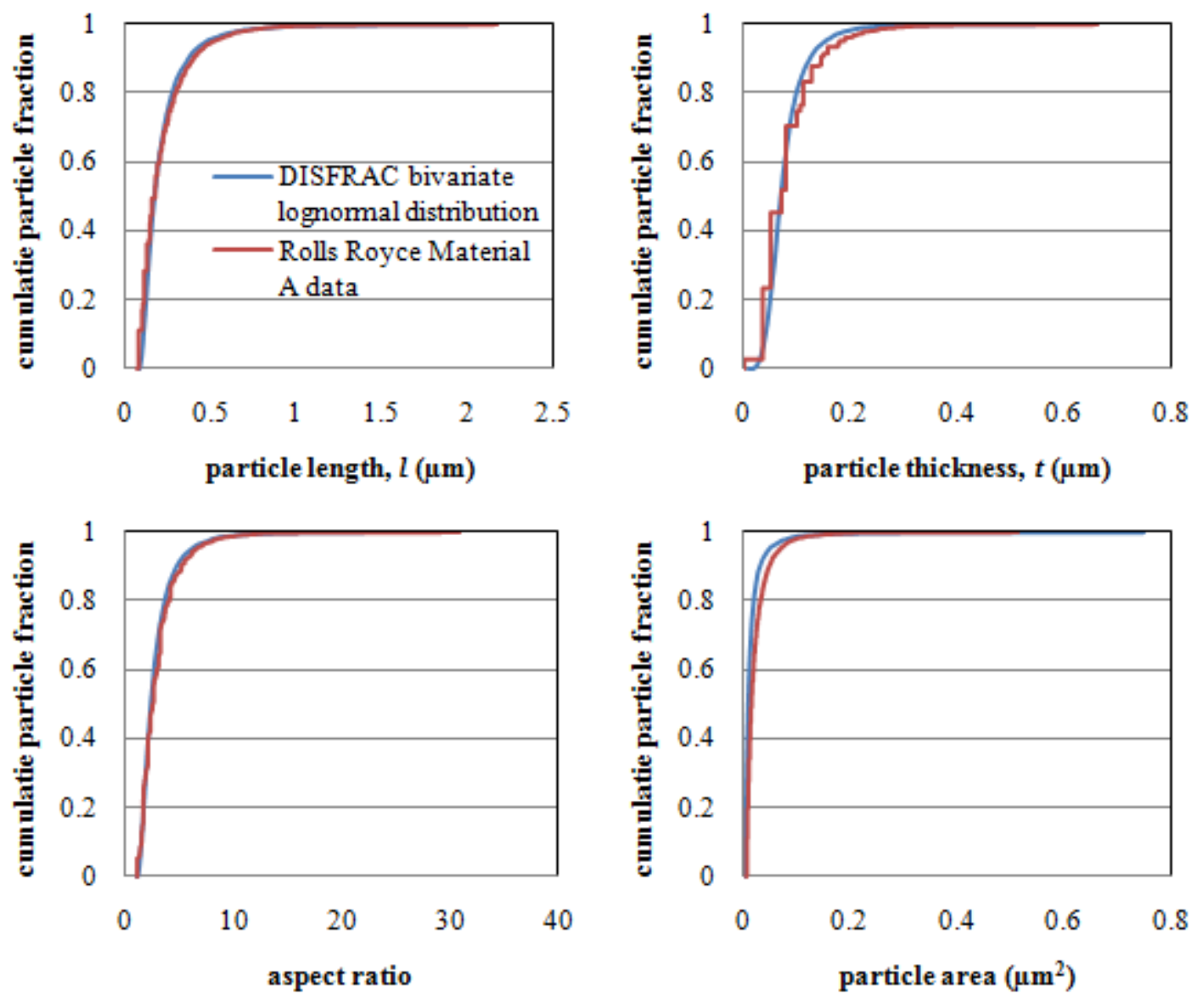

Figure 4. Cumulative distributions of various particle attributes. Experimental data and derived distribution output shown, each for 5628 particles.

\subsubsection{Grain size}

For a Monte Carlo simulation, the grain size is selected randomly from a user selected distribution. The available distributions are described below.

\subsubsection{Rolls Royce Material A 2012 (g_size $=1)$}

The data from Rolls Royce for this material provided grain diameter, area, and aspect ratio information for 9827 grains. DISFRAC currently requires only an average diameter measurement for each grain. A 3-parameter Weibull distribution (Eqn. 7) was fit to the Rolls Royce data. Some of the statistics on the grain size distribution and the calibrated parameters can be found in Table 4. Figure 5 presents the cumulative distribution and probability density for both experimental data and DISFRAC output for the same number of particles.

$$
P=1-\exp \left[-\left(\frac{x-a}{b}\right)^{c}\right]
$$


DRAFT

Table 4. Grain size statistics and Weibull parameters for Rolls Royce Material A

\begin{tabular}{|l|l|l|l|l|l|l|}
\hline & avg. \pm std. $\operatorname{dev}(\boldsymbol{\mu m})$ & $\max (\boldsymbol{\mu m})$ & $\min (\boldsymbol{\mu m})$ & a & b & c \\
\hline grain diameter & $4.3 \pm 6.6$ & 86 & 0.74 & 0.73 & 1.95 & 0.5 \\
\hline
\end{tabular}
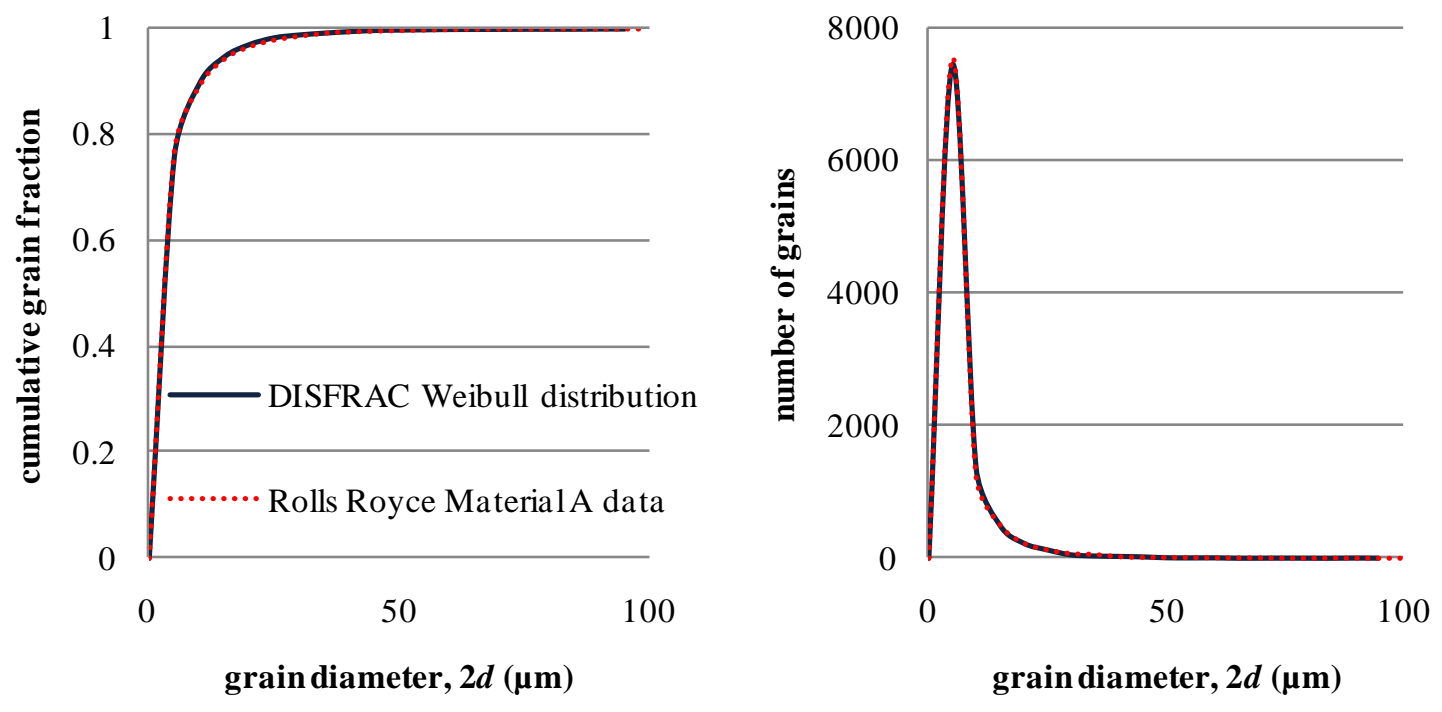

Figure 5. Cumulative and density distributions for grain diameter. Experimental data and derived distribution output shown, each for 9827 grains. 


\section{DISFRAC OUTPUT}

DISFRAC writes up to 3 user-selected output files, as described below.

\subsection{SUMMARY FILE}

The file summary_results.out contains compiled results from the trials at each temperature. For each temperature, this file records the number of trials for each termination code (see below) and the minimum toughness corresponding to the various particle fracture outcomes (non-negative termination codes). This file also contains an echo of the input file.

Table 5. Termination codes

\begin{tabular}{|l|l|}
\hline termination code & definition \\
\hline 0 & particle fractures but microcrack does not enter first grain \\
\hline 1 & microcrack arrests at grain boundary before specimen failure \\
\hline 2 & not currently used \\
\hline 3 & microcrack runs through max. \# grains \\
\hline 4 & microcrack exits plastic zone without arresting \\
\hline-1 & void forms before particle fractures \\
\hline-2 & particle does not fracture below max allowed load \\
\hline-3 & crack initiates outside plastic zone \\
\hline-4 or -5 & numerical problems \\
\hline
\end{tabular}

\subsection{ALL RESULTS}

This file includes all geometric information and significant amounts of calculated variables used in the various energy balances. This file gets very large but contains a great deal of information useful for debugging or analyzing trends and behaviors. For each trial, data is written for the first 21 columns (data pertaining to crack initiation model). For trials for which the particle fractures, further data is written for each grain boundary calculation. Table 6 lists the columns in this output file.

\subsection{FAILED PARTICLE RESULTS}

This file contains only the inputs (geometric data, temperature) and major result quantities for particles in which a microcrack initiates (termination code $>-1$ ). The column headings follow Table 6 , but omit the intermediate calculation results. This file is useful for assessing trends between toughness and the various geometric quantities. It is generally much smaller, and thus more tractable to import into a spreadsheet, than the all_results file. 
Table 6: Columns in all_results.out. Columns 21-30 are repeated for each grain boundary at which an energy balance is computed, see Figure 6.

\begin{tabular}{|c|c|c|c|}
\hline & Column label & units & definition \\
\hline 1 & Case & none & trial number at current temperature (sequential) \\
\hline 2 & Temperature & ${ }^{\circ} \mathrm{C}$ & Temperature for current trial (user-defined) \\
\hline 3 & xpos & $\mu \mathrm{m}$ & $\mathrm{x}$-coordinate of particle, relative to macrocrack tip \\
\hline 4 & ypos & $\mu \mathrm{m}$ & y-coordinate of particle, relative to macrocrack tip \\
\hline 5 & $\mathrm{~h}$ & $\mu \mathrm{m}$ & radial distance from macrocrack tip to particle \\
\hline 6 & omega & o & angle between macrocrack plane and particle position \\
\hline 7 & 1 & $\mu \mathrm{m}$ & length of particle (2x major axis length) \\
\hline 8 & asp.ratio & none & aspect ratio of particle (major axis/minor axis) \\
\hline 9 & alpha & $\circ$ & $\begin{array}{l}\text { orientation of particle; angle between macrocrack plane and particle } \\
\text { major axis }\end{array}$ \\
\hline 10 & eta & $\circ$ & $\begin{array}{l}\text { orientation of particle cleavage plane, with respect to macrocrack } \\
\text { plane }\end{array}$ \\
\hline 11 & $2 \mathrm{r}$ & $\mu \mathrm{m}$ & $\begin{array}{l}\text { particle crack length (accounts for particle and cleavage plane } \\
\text { orientation; this is not } 2 x \text { minor axis) }\end{array}$ \\
\hline 12 & sigma_c & $\mathrm{MPa}$ & $\begin{array}{l}\text { critical stress (applied remote normal stress at particle fracture). Set } \\
\text { to zero for non-fracture outcomes. }\end{array}$ \\
\hline 13 & Kc & $\mathrm{MPa} \sqrt{\mathrm{m}}$ & fracture toughness for trial. Set to zero for non-fracture outcomes. \\
\hline 14 & code & none & $\begin{array}{l}\text { termination code (indicates outcome of trial). See Table } 5 \text { for specific } \\
\text { definitions. }\end{array}$ \\
\hline 15 & num_GB & none & $\begin{array}{l}\text { number of grain boundaries across which microcrack successfully } \\
\text { propagates. }\end{array}$ \\
\hline 16 & sigma_y & $\mathrm{MPa}$ & yield stress of material at trial temperature \\
\hline 17 & tauf & $\mathrm{MPa}$ & friction stress of material at trial temperature \\
\hline 18 & F_SB & $\mathrm{J} / \mathrm{m}^{2}$ & resistance from localized grain plasticity on particle crack initiation \\
\hline 19 & U_MBL & $\mathrm{J} / \mathrm{m}^{3}$ & strain energy density acting on particle \\
\hline 20 & obj_fun & $\mathrm{J} / \mathrm{m}^{2}$ & phase 0 (particle crack initiation) objective function value \\
\hline $21 *$ & $2 \mathrm{~d}$ & $\mu \mathrm{m}$ & slip band length (grain size) \\
\hline $22 *$ & theta & $\circ$ & orientation of slip band with respect to macrocrack plane \\
\hline $23 *$ & x_gb & $\mu \mathrm{m}$ & $\mathrm{x}$-coordinate of grain boundary $i$ at cleavage plane intersection point \\
\hline $24 *$ & y_gb & $\mu \mathrm{m}$ & y-coordinate of grain boundary $i$ at cleavage plane intersection point \\
\hline $25 *$ & twist & o & twist angle for grain boundary $i$ \\
\hline $26 *$ & LHS & $\mathrm{J} / \mathrm{m}^{2}$ & left-hand-side (driving term) of grain boundary $i$ energy balance \\
\hline $27 *$ & RHS & $\mathrm{J} / \mathrm{m}^{2}$ & right-hand-side (resistance term) of grain boundary $i$ energy balance \\
\hline $28 *$ & R_D & $\mathrm{J} / \mathrm{m}^{2}$ & grain plasticity resistance term $i$ \\
\hline $29 *$ & gamma_GB & $\mathrm{J} / \mathrm{m}^{2}$ & grain boundary tearing resistance term $i$ \\
\hline $30 *$ & eps_pl & none & plastic strain at grain boundary $i$ \\
\hline
\end{tabular}




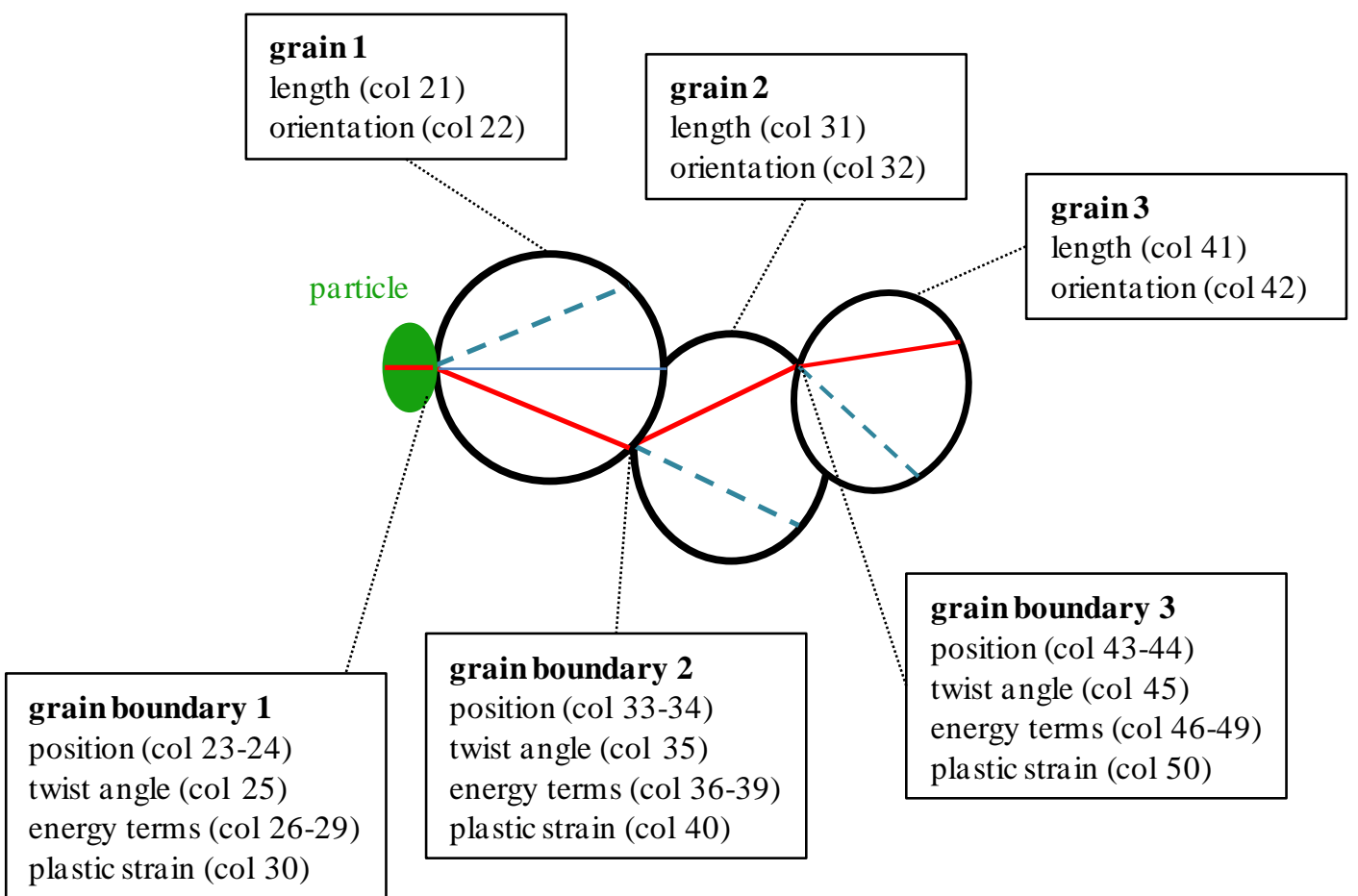

Figure 6. Definition of repeating columns in all_results output file 


\section{PARTICLE CRACK INITIATION MODEL}

The particle crack initiation model determines the applied load required to fracture a carbide particle embedded in a ferrite matrix in the plastic zone of a nearby macrocrack. In this model, the particle's size, shape, location, the local grain structure, and the macroscopic stress-strain fields influence the propensity for a particle to fracture. The following sections discuss the theory, implementation, and some verification of the crack initiation model in DISFRAC.

\subsection{MODEL DEFINITION}

This current version of the particle crack initiation model, version p0_e2, determines the applied load required to crack a specified particle. This model employs an energy approach based on the total energy required (and available) to crack the particle. This is somewhat different from a Griffith-style energy balance which assesses the energy change for an incremental increase in crack length. The particle is initially uncracked but has a pre-defined plane on which a crack may potentially form. The only materialbased failure property required is surface energy; this was one of the motivations for this approach. Ultimate strength and/or fracture toughness values of carbide particles are difficult to obtain while surface energy is comparatively well-known.

This model represents the follow behavior.

1. Loading (energy available to create the crack) relies only on the macroscropic stress-strain fields at the particle location not localized dislocation-based effects.

2. The macroscopic stress-strain fields (from the elastic-plastic finite element MBL solutions) contribute to the load via the strain energy density in the matrix, at the particle location.

3. The strain energy density calculation uses only stress and plastic strain invariants (von Mises stress, equivalent plastic strain). This is equivalent to full tensor-based strain energy calculation provided the elastic strain is insignificant compared to the plastic strain.

4. The strain energy decreases monotonically with distance from the macrocrack tip. This is in contrast to the normal stress (relative to the macrocrack), which develops a peak at some (small) distance from the macrocrack tip. Thus this model will predict different patterns of cracking incidence compared to normal stress-based models.

5. The driving energy is independent of the grain size and orientation.

6. The surface energy defines the minimum energy required to break the particle.

7. Grain-based slip resists crack initiation. The amount of resistance depends on the friction stress, the grain orientation, and the grain size.

a. Resistance increases with increasing grain size.

b. Resistance increases with increasing temperature (decreasing friction stress).

c. Resistance depends nonlinearly on grain orientation.

8. Resistance is independent of particle size and orientation.

\subsubsection{Objective}

The objective of the model is to find the applied load such that the energy balance-based objective function (Eqn. 8) is satisfied. 


$$
\Theta\left(\sigma_{\text {applied }}\right)=U_{M B L}\left(\epsilon_{p}\right) \frac{V_{p}}{A_{\text {pcrack }}}-\left(2 \gamma_{c}+F_{S B}\right)=0
$$

$U_{M B L}$ is a measure of strain energy available to drive crack growth; it depends only on the current equivalent plastic strain and the parameters of the material model. $V_{p}$ is the volume of the particle and $A_{\text {pcrack }}$ is the surface area of the particle crack plane. The resistance terms include the surface energy of carbide $\left(\gamma_{c}\right)$ and a localized grain-based dissipative term, $F_{S B}$.

\subsubsection{Derivation}

The objective function above results from equating the energy available to crack the particle to the energy expended in the creation of the crack. Equation 9 illustrates this energy balance, with the total driving energy on the left and the two resistance terms on the right.

$$
U_{M B L}\left(\epsilon_{p}\right) V_{p}=2 \gamma_{c} A_{\text {pcrack }}+F_{S B} A_{\text {pcrack }}
$$

The energy available to create the crack comes from the strain energy imparted to the carbide by the deforming matrix. $U_{M B L}\left(\epsilon_{p}\right)$ is the strain energy density and $V_{p}$ is the particle volume; the product of $U_{M B L}$ and $V_{p}$ is the total strain energy in the particle. $F_{S B}$ represents the net shielding force (per unit dislocation length) from the dislocation pileup on the slip band in the adjacent grain and $\gamma_{c}$ is the surface energy density of the particle material. The following sections explain the meaning and calculation of the strain energy and slip band terms. The surface energy term $\left(2 \gamma_{c} A_{\text {pcrack }}\right)$ is simply the total amount of energy needed to create two surfaces (one for each face of the crack) to fully crack the particle. Currently, $\gamma_{\mathrm{c}}=2.0 \mathrm{~J} / \mathrm{m}^{2}$, the lower end of the range of carbide surface energies predicted by Chiou Jr. and Carter8.

\subsubsection{Strain energy}

The strain energy in an elastic carbide within a plastically deforming ferrite matrix cannot be expressed analytically in general. The approach employed in DISFRAC equates the strain energy density of the carbide to an approximate measure of strain energy density in the matrix. This model assumes that the strain energy imparted to the particle is equivalent to the strain energy of an equivalent volume of matrix material.

The evolution of the stress and strain fields in the matrix material, as quantified by the finite element model and the Zerilli-Armstrong material model, leads to strain energy accumulation with increased remote loading. The stress and strain fields are, of course, tensors that evolve nonlinearly with increasing remote load.

To compute the strain energy precisely, using the actual tensors and accounting for elastic and plastic strains, would require a numerical integration over time (load) and space either within DISFRAC or stored from ABAQUS. An approximate measure of strain energy is defined instead employing the following assumptions.

\section{Assumptions}

1. The stress and strain fields are essentially constant over the small area defining the particle. Thus the volume integral drops out; the strain energy density can be multiplied by the particle volume to find the total strain energy. 


\section{DRAFT}

2. The von Mises stress and equivalent strain rather than the actual tensor components are sufficient to compute the strain energy density.

3. The plastic strains are much larger than the elastic strains; the elastic contribution to the ferrite matrix strain energy is thus neglected.

The above assumptions reduce the integrals over space and strain increment, and summation across tensor components to a single integral of scalar components as shown in Eqn. 10. Here, $E$ indicates the total strain energy in a region of volume $\mathrm{V}$.

$$
E=\int\left(\sum_{j=1}^{3} \sum_{i=1}^{3} \int \sigma_{i j} d \epsilon_{i j}\right) d V \approx V \int \sigma_{M i s e s} d \epsilon_{p}
$$

Equation 11 defines the strain energy density, $U_{M B L}$ as employed in DISFRAC. It computes the area under the stress-strain curve defined by the Zerilli-Armstrong constitutive relation up to a given strain level, $\epsilon_{p}$. This is the effective plastic strain corresponding to current von Mises equivalent stress determined via the finite element analyses for the particle location and the applied load. Figure 7 illustrates the parameters and calculated area.

$$
U_{M B L}=\int_{0}^{\epsilon_{p}} \sigma_{Z A}\left(\epsilon_{Z A}\right) d \epsilon_{Z A}
$$

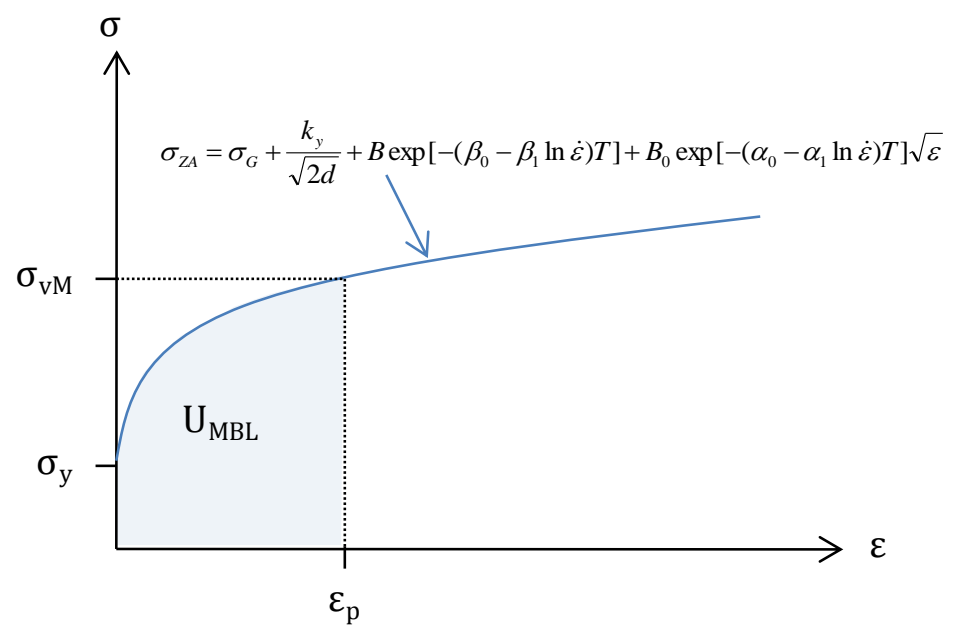

Figure 7. Strain energy definition for Zerilli-Armstrong model

The form of the Zerilli-Armstrong model allows the strain energy density integral to be computed analytically;

$$
U_{M B L}=\left[\sigma_{G}+k_{y} / \sqrt{2 d}+B e^{-\left(\beta_{0}-\beta_{1} \ln \dot{\epsilon}\right) T}\right] \epsilon_{p}+B_{0} e^{-\left(\alpha_{0}-\alpha_{1} \ln \dot{\epsilon}\right) T} \frac{1}{2} \epsilon_{p}^{3 / 2}
$$




\section{DRAFT}

\subsubsection{Dislocation mechanics}

Within a grain, dislocation motion occurs along specific slip planes, based on the grain orientation. The dislocations move when the local shear stress on the slip plane $\left(\tau_{\theta}\right)$ exceeds the friction stress $\left(\tau_{f}\right)$ of the material. Dislocation movement may be blocked by obstacles such as grain boundaries and particles. Dislocations then pile up at the obstacles.

DISFRAC aggregates the pileups in a given particle with a single slip band comprised of glide edge dislocations following the techniques of Yokobori and colleagues ${ }^{9-12}$. DISFRAC employs a distribution of infinitesimal dislocations, rather than discrete dislocations. Following elastic dislocation theory, the shear stress at a point $x$ from a glide dislocation at point $\xi$ ( $x$ and $\xi$ on the plane of the slip band) is ${ }^{13}$ :

$$
\tau_{\text {dislocation }}=\frac{G b}{2 \pi(1-v)} \frac{1}{x-\xi}
$$

$G$ is the shear modulus, $b$ is the Burgers vector, and $v$ is Poisson's ratio. Equilibrium of the dislocation distribution (described by $f(\zeta)$ ) with the effective shear stress requires

$$
\tau_{\theta}(x)-\tau_{f}=\int_{-d}^{d} \frac{G b}{2 \pi(1-v)} \frac{1}{x-\xi} f(\xi) d \xi
$$

where $\tau_{\theta}(x)$ is the shear stress applied to the dislocation distribution by the background material.

Assuming the background shear stress is essentially constant along the slip band (i.e., $\tau_{\theta}(x)=\tau_{\theta}$ ), a Hilbert transform provides the solution as

$$
f_{S B}(x)=\frac{\tau_{e f f}}{\pi A} \frac{x}{\sqrt{d^{2}-x^{2}}}
$$

where $\tau_{\text {eff }}=\operatorname{sgn}\left(\tau_{\theta}\right)\left[\max \left(\left|\tau_{\theta}\right|-\tau_{f}, 0\right)\right]$ and $A=G b /[2 \pi(1-v)]$.

This result is equivalent to the slip band acting as a linear-elastic mode II crack. The stress field near the tip of the slip band can thus be characterized by a mode II stress intensity factor, $K_{I I s}$;

$$
K_{I I s}=\sqrt{\pi d} \tau_{e f f}
$$

The slip band dislocations create a force per unit dislocation length that acts on dislocation ahead of the tip of the pileup ${ }^{13}$. This force, $F_{S B}=K_{I I S}^{2} / E^{\prime}$, is identical to the definition of energy release rate $G$ for the growth of a crack equivalent to the slip band. $E^{\prime}$ is the plane strain elastic modulus. Thus,

$$
F_{S B}=\frac{\pi \tau_{e f f}^{2} d^{2}}{E^{\prime}}
$$


This force is then multiplied by the area of the potential particle crack $\left(A_{\text {pcrack }}\right)$, to provide the local dislocation-based resistance to particle crack formation. Multiplication by this area provides the correct units and simplifies the contribution of the particle shape in the model to just the ratio of particle volume to particle crack plane area. It can be rationalized by considering it the energy that would have been released if the slip band extended across the particle, rather than being blocked by it. Initial results indicate that this is a numerically reasonable plastic work term; nevertheless it is a candidate for further study and revision.

\subsection{IMPLEMENTATION}

\subsubsection{Algorithm}

DISFRAC determines the load for which $\Theta\left(\sigma_{\text {applied }}\right)=0$ (Eqn. 8) by first bracketing the solution by a simple load ramping process, and then applying the Regula-Falsi method to compute the critical load. For a given estimate of the applied load, the follow sequence of calculations is performed.

1. Compute the stress components $\left(\sigma_{x x}, \sigma_{y y}, \sigma_{x y}\right)$ and invariants $\left(\sigma_{\text {Mises, }} \sigma_{\text {mean }}\right)$ at the particle position for the current global $J$ level by interpolating from the relevant finite element MBL results

2. Compute the effective plastic strain $\left(\varepsilon_{p}\right)$ from the von Mises stress $\left(\sigma_{\text {Mises }}\right)$ using the ZerilliArmstrong equation

3. Compute the shear stress $\left(\tau_{\theta}\right)$ acting on the slip band from the MBL stress components (tensor rotation from global to local coordinate system)

4. Compute the effective stress acting on the slip band dislocations, using the temperaturedependent friction stress: $\tau_{\text {eff }}=\operatorname{sgn}\left(\tau_{\theta}\right)\left[\max \left(\left|\tau_{\theta}\right|-\tau_{f}, 0\right)\right]$

5. Compute the stress-intensity factor for the slip band: $K_{I I S}=\sqrt{\pi d} \tau_{\text {eff }}$

6. Compute $F_{S B}=K_{I I S}^{2} / E^{\prime}$

7. Compute the strain energy density from

$$
U_{M B L}=\left[\sigma_{G}+k_{y} / \sqrt{2 d}+B e^{-\left(\beta_{0}-\beta_{1} \ln \dot{\epsilon}\right) T}\right] \epsilon_{p}+B_{0} e^{-\left(\alpha_{0}-\alpha_{1} \ln \dot{\epsilon}\right) T} \frac{1}{2} \epsilon_{p}^{3 / 2}
$$

using $\varepsilon_{p}$ from step 2

8. Compute the particle volume to area ratio, $V_{p} / A_{\text {pcrack }}$ (see section 4.2.2)

9. Compute the current value of the objective function (Eqn. 8) using the results in steps 6, 7, 8, and the material constant surface energy, $\gamma_{c}$

DISFRAC caps the applied stress such that $\max \left[K_{\text {applied }}\right]=200 \mathrm{MPa} \sqrt{\mathrm{m}}$. If the equivalent plastic strain $\varepsilon_{p}$ exceeds a limiting value (void strain) before particle crack initiation occurs, DISFRAC assumes a void is formed and eliminates this particle from further consideration. Currently, the void strain is $\epsilon_{n}=0.04$ as in Xia and Chen7.

\subsubsection{Particle size and orientation}

The particle crack initiation model directly needs only the ratio of particle volume to the area of the potential crack plane; i.e., $V_{p} / A_{\text {pcrack }}$. Nevertheless, this ratio depends on particle size, shape, orientation with respect to the macrocrack plane and orientation of the potential crack plane.

Figure 8 shows the relevant geometry. 


\section{DRAFT}

\subsubsection{Assumptions and definitions}

1. Particle is an ellipsoid with two equal length axes $(a)$.

2. The third axis (major axis) has length $b$ with $b \geq a$.

3. The particle's orientation with respect to the global coordinate system is given by the angle $(\alpha)$ between the particle's major axis and the global x-direction.

4. The particle orientation is random and characterized by a uniform distribution with $0 \leq \alpha<$ $180^{\circ}$.

5. The potential crack plane in the particle is oriented angle $\eta$ from the global x-direction.

6. The potential crack plane orientation is random and characterized by a uniform distribution with $-15^{\circ} \leq \eta<15^{\circ}$.

7. No out-of-plane rotation is considered (all rotation is about global z-axis).

8. The potential crack plane is an ellipse with long axis $b_{\alpha \eta}$ and short axis $a$.

a

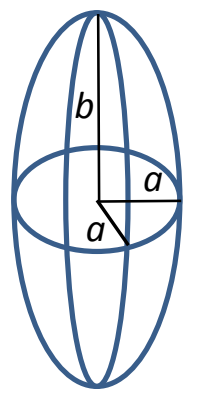

b

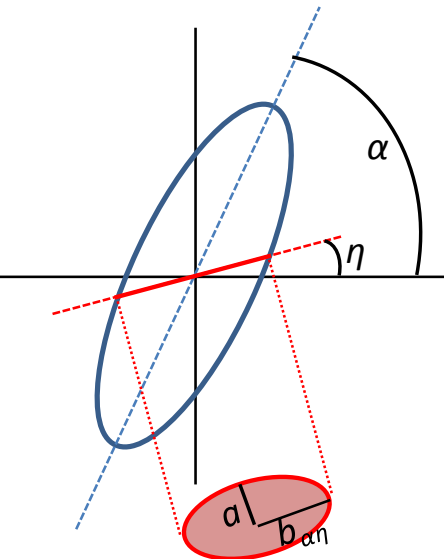

Figure 8. Particle shape and orientation definitions. (a) ellipsoidal particle, with major axis $=2 b$ and equal minor axes $=2 a$, (b) particle and potential crack plane orientation with respect to global coordinate system. Elliptic potential crack plane shown in red.

The long axis of the potential crack plane can be computed as

$$
b_{\alpha \eta}=\frac{a b}{\sqrt{[a \cos (\alpha-\eta)]^{2}+[b \sin (\alpha-\eta)]^{2}}}
$$

Substituting the aspect ratio $a_{r}=b / a \geq 1$

$$
b_{\alpha \eta}=\frac{b / a_{r}}{\sqrt{\frac{1}{a_{r}^{2}}[\cos (\alpha-\eta)]^{2}+[\sin (\alpha-\eta)]^{2}}}
$$

- For the case of a spherical particle $\left(a=b, a_{r}=1\right), b_{\alpha \eta}=b$ as expected. 
- The volume of the ellipsoid is independent of orientation and computed as $V_{p}=\frac{4}{3} \pi a^{2} b$.

- The area of the potential crack ellipse shape is $A_{\text {pcrack }}=\pi a b_{\alpha \eta}$.

Thus the volume to crack plane area ratio for the rotated particle is

$$
\frac{V_{p}}{A_{\text {pcrack }}}=\frac{4}{3} b \sqrt{\frac{1}{a_{r}^{2}}[\cos (\alpha-\eta)]^{2}+[\sin (\alpha-\eta)]^{2}}
$$

\subsubsection{Simple checks and observations}

1. Spherical particle $\left(a_{r}=1\right)$ :

$$
\frac{V_{p}}{A_{\text {pcrack }}}=\frac{4}{3} b
$$

2. Major axis aligned with global $y$-axis, crack plane aligned with global $x$-axis $\left(\alpha=90^{\circ}, \eta=0^{\circ}\right)$ :

$$
\frac{V_{p}}{A_{\text {pcrack }}}=\frac{4}{3} b
$$

3. Major axis aligned with global $x$-axis, crack plane aligned with global $x$-axis $\left(\alpha=0^{\circ}, \eta=0^{\circ}\right)$ :

$$
\frac{V_{p}}{A_{\text {pcrack }}}=\frac{4}{3} \frac{b}{a_{r}}
$$

4. The maximum value $\left(V_{p} / A_{\text {pcrack }}=1.33\right)$ occurs when the major axis and potential crack plane are perpendicular, $\alpha-\eta=90^{\circ}$, and is independent of aspect ratio.

5. For non-perpendicular orientations, increasing the aspect ratio decreases $V_{p} / A_{p c r a c k}$, and thus decreasing the net loading on the particle. Increasing $a_{r}$ from 2 to 10 decreases the minimum normalized volume/area ratio from 0.667 to 0.133 .

6. $V_{p} / A_{\text {pcrack }}$ tends toward minimum values as $(\alpha-\eta)$ tends toward $0^{\circ}$ or $180^{\circ}$, i.e. when the minor axis and potential crack plane align.

\subsection{VERIFICATION}

\subsubsection{Strain energy assumptions}

The strain energy density calculation employs only the von Mises and equivalent plastic strain instead of the full stress tensor and the full total strain tensor. Figure 9 demonstrates the suitability of this approximation. The inner product of the stress and total strain (in blue) is nearly indistinguishable from the product of the von Mises stress and equivalent plastic strain (in red) over a large strain range. Thus the strain energy density should also be essentially identical for the exact and approximate computations. 


\section{DRAFT}

The strain energy increases monotonically with proximity to the crack tip, for both the exact and approximate computations. Thus, with all else constant, particles closer to the crack tip fracture more easily (i.e., at lower load). This is not necessarily the case for a stress-based criterion (maximum normal stress or maximum principal stress). In a geometrically nonlinear analysis of the stress fields near a crack, tensile stresses reach a maximum value at a finite distance from the crack tip. A stress-based fracture criterion should reveal preferred particle locations for crack initiation. Experimental evidence in support of both trends exists however, and a goal for future work is to revisit this issue when new data becomes available.

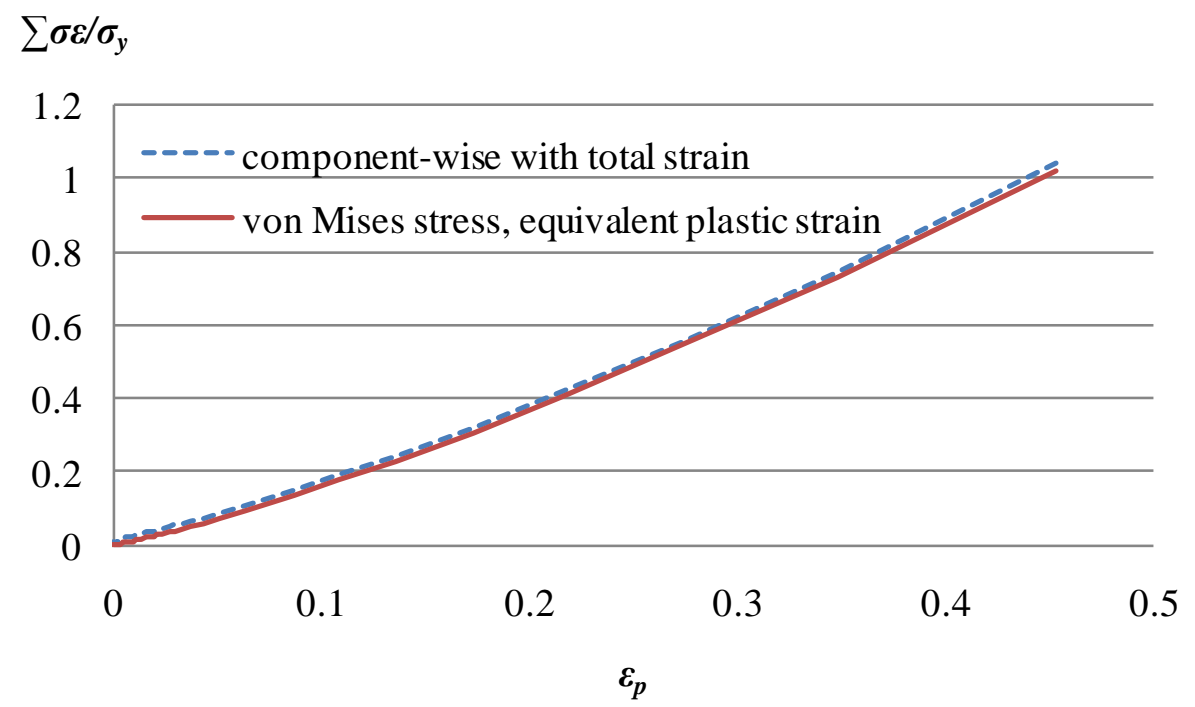

Figure 9. Assessing the accuracy of the von Mises and plastic equivalent strain method for computing strain energy density. Data shown corresponds to $T=100^{\circ} \mathrm{C}, \omega=45^{\circ}$, and $h=100 \mu \mathrm{m}$. 


\section{TRANSGRANULAR PROPAGATION MODEL}

If a crack initiates in a given particle, this microcrack becomes a candidate for causing cleavage fracture by propagating through the surrounding grains. DISFRAC assumes the crack arrests momentarily at each grain boundary such that local dislocation pileups occur under static equilibrium conditions. The crack will then propagate into the next grain, if it is energetically favorable to do so.

The transgranular propagation model is based on the Griffith energy balance method. Griffith asserted that a crack will grow if doing so means the total energy of the specimen decreases. Let the crack area be $A=2 a B$, where $B$ is the thickness and $a$ is the $1 / 2$ crack length. Then, the crack grows if

$$
\frac{d E}{d A}=\frac{d \Pi}{d A}+\frac{d W}{d A}<0
$$

$E$ is the total energy, $\Pi$ is the potential energy (from external forces and internal strain energy), and $W$ is work required to grow the crack. The follow sections defines the terms and calculations DISFRAC employs to quantify the change in energy as the microcrack grows across a grain boundary. The last section discusses the manifestation of crack growth in DISFRAC.

\subsection{ENERGY RELEASE RATE}

The decrease in potential energy required for the energy balance assessment is equivalent to Griffith's energy release rate $G$, also sometimes referred to as the driving force for crack growth. In DISFRAC, the sharp microcrack releases energy as it grows. At the grain boundary, DISFRAC assumes the crack temporarily arrests; inertial effects are not considered. To cross the grain boundary, the crack must change directions to the cleavage plane in the adjacent grain. Thus, the energy release rate depends on the mode I and mode II stress-intensity factors of the right-hand microcrack tip and the angle between the current and subsequent cleavage planes (tilt angle, $\Delta \theta$ ).

Appendix A discusses the relationship between stress-intensity and energy release rate, including the techniques for accounting for crack direction change. Let $\alpha_{i j}$ be the angle between adjacent grain cleavage planes (as in Figure 10), then the energy release rate is

$$
G\left(\alpha_{i j}\right)=\frac{k_{I}^{2}\left(\alpha_{i j}\right)+k_{I I}^{2}\left(\alpha_{i j}\right)}{E^{\prime}}
$$




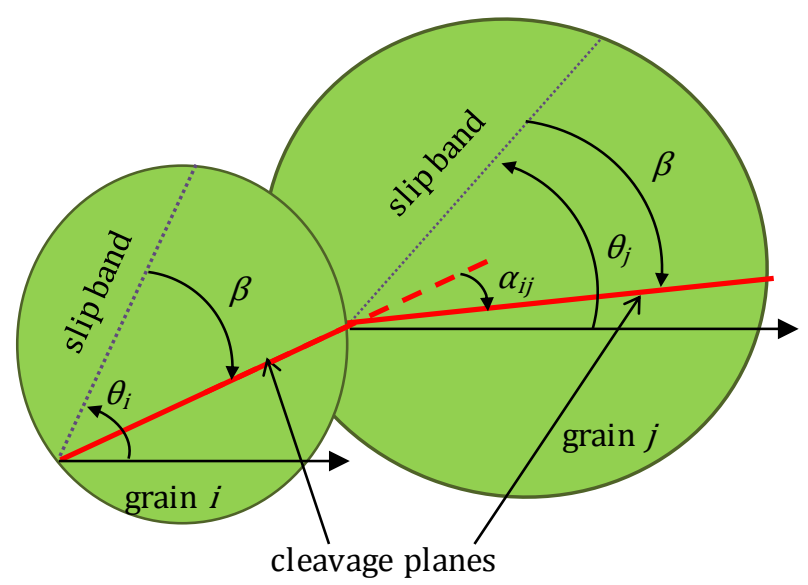

Figure 10. Angle between adjacent cleavage planes. $\boldsymbol{\alpha}_{\boldsymbol{i} j}=\boldsymbol{\theta}_{\boldsymbol{j}}-\boldsymbol{\theta}_{\boldsymbol{i}}$, clockwise positive. As shown, $\boldsymbol{\alpha}_{\boldsymbol{i} \boldsymbol{j}}$ is negative.

The local stress intensity factors, $k_{I}$ and $k_{I I}$, depend on the microcrack stress intensity factors ( $K_{I r}$ and $K_{I I r}$, refer to Section 7) as

$$
\begin{gathered}
k_{I}\left(\alpha_{i j}\right)=\max \left[0, C_{11}\left(\alpha_{i j}\right) K_{I r}+C_{12}\left(\alpha_{i j}\right) K_{I I r}\right] \\
k_{I I}\left(\alpha_{i j}\right)=\left[C_{21}\left(\alpha_{i j}\right) K_{I r}+C_{22}\left(\alpha_{i j}\right) K_{I I r}\right]
\end{gathered}
$$

The correlation coefficients $\left(C_{11}, C_{12}, C_{21}, C_{22}\right)$ are defined in Eqn. A.5. Negative values for the local mode I stress-intensity are not allowed as this would correspond to interpenetration of the crack faces. The local mode II stress-intensity may be either positive or negative; both represent physically acceptable conditions.

\subsection{RESISTANCE TO CRACK GROWTH}

\subsubsection{Plastic work/dislocation shielding}

The material near the microcrack tip resists the crack growth. There are many views of how to describe and quantify this contribution to the overall energy of a system. Most common is an empirical plastic work term, $w_{p}$, defined as the plastic work done per unit area of new surface.

DISFRAC assumes that the microcrack arrests at a grain boundary and the dislocation distributions representing the microcrack and slip band are in static equilibrium with each other and the applied load. Thus, during this pause in crack growth, the dislocations in the slip band are not in motion once equilibrium is established. Without dislocation motion, work is not actually occurring. Instead, DISFRAC takes the following approach to quantifying the resistance to crack growth from nearby plasticity.

The glide dislocation distribution of the slip band is essentially equivalent to a linear-elastic mode II crack; the stress and displacement fields near the slip band tips may be represented by the mode II Kfields of linear elastic fracture mechanics (LEFM). The dislocation crack extension force described by Weertman ${ }^{13}$ for such a dislocation pileup is identical to the energy release rate of Griffith: $F_{E}=G=\frac{K_{I I}^{2}}{E^{\prime}}$. However, the grain boundary and the impinging microcrack prevent extension of the slip band. The slip 
band extension force must then be transferred to dislocations surrounding the slip band tip ${ }^{13}$. This term has units of force per length; i.e. the force per unit slip band thickness.

DISFRAC assumes that this force is transferred to dislocations along the cleavage plane candidate for microcrack extension (i.e., within the same grain as the slip band). To resolve the force directed along the slip band axis to the cleavage plane, DISFRAC follows the techniques in Appendix A for computing effective $\mathrm{k}$-factors for change in crack direction. The angle between the slip band axis (positive away from the slip band) and the cleavage plane is fixed at $135^{\circ}$ for the BCC grain structure. The mode II stress intensity factor of the slip band produces both mode I and mode II factors on the cleavage plane:

$$
\begin{aligned}
& k_{I}\left(135^{\circ}\right)=C_{12}\left(135^{\circ}\right) K_{I I d}=-0.4059 K_{I I d} \\
& k_{I I}\left(135^{\circ}\right)=C_{22}\left(135^{\circ}\right) K_{I I d}=-0.5972 K_{I I d}
\end{aligned}
$$

The resistance of the grain dislocations to the microcrack advance is thus

$$
R_{D}=\frac{k_{I}^{2}+k_{I I}^{2}}{E^{\prime}}=\frac{0.52 K_{I I d}^{2}}{E^{\prime}}
$$

This method assumes that the dislocation effect is always shielding. Thus the effective energy release rate for the microcrack, accounting for tilt direction change and localized plastic dissipation is

$$
G_{e f f}=G_{i j}-R_{D}
$$

\subsubsection{Grain misorientation}

When a cleavage crack passes from one grain into another, it must cross the grain boundary. The misorientation of two adjacent grains, for the purposes of DISFRAC, is characterized by the tilt and twist angles. The tilt angle, $\Delta \theta$, describes the in-plane angle change between the adjacent grains and the twist angle describes the out-of-plane angle change between the adjacent grains, see Figure 11. In DISFRAC, the effects of tilt and twist are treated separately. The driving force of the energy balance considers the energy release rate of the microcrack tip for an incremental increase in crack length along the new cleavage plane, accounting for tilt angle.

The twist angle, however, requires a separate treatment. The cleavage planes in adjacent grains only meet at distinct points. Between these points lie "persistent grain boundary areas", following the observations of Qiao et al. ${ }^{19-21}$. As the cleavage crack passes from one grain to another, these persistent areas must separate before the crack can propagate across the grain.

DISFRAC's approach considers the energy expended to create the new surfaces from the persistent boundary. In the process of fully separating the grain boundary areas, the cleavage crack advances by $\delta_{g b}$, the distance required to reduce the traction between adjacent surfaces to zero, see Figure 12. The existing microcrack in grain A has an effective energy release rate, $G_{\text {eff. }}$ Thus energy released as the cleavage microcrack advances $\delta_{g b}$ is approximately $G_{e f f} \delta_{g b} B$, where $B$ is the thickness. The energy required to separate the cleavage surfaces is $2 \gamma_{s} \delta_{g b} B$ and the energy required to separate the persistent boundary surfaces is $2 \gamma_{s, g b} A_{\text {pers }}$, where $\gamma_{s}$ is the surface energy of cleavage plane, $\gamma_{s, g b}$ is the surface energy of the grain boundary plane and $A_{\text {pers }}$ is the persistent boundary area. Thus the microcrack will propagate across the grain boundary if 
DRAFT

$$
G_{e f f} \delta_{g b} B>2 \gamma_{s} \delta_{g b} B+2 \gamma_{s, g b} A_{p e r s}
$$

(Note that the plastic work is included in the $G_{\text {eff }}$ term).

The persistent boundary area depends on the number of breakthrough points, the width of the boundary, and the twist angle. Figure 13. Persistent grain boundary area for multiple breakthrough points illustrates the grain boundary area for multiple breakthrough points. For a single breakthrough point, the associated area is $A_{p b t}=\left(\frac{d_{b}}{n}\right)^{2} \frac{\tan \phi}{1+\tan ^{2} \phi}$, where $n$ is the number of breakthrough points along the grain boundary of width $2 d_{b}$. Thus, for $n$ breakthrough points;

$$
A_{\text {pers }}=\frac{d_{b}^{2}}{n}\left(\frac{\tan \phi}{1+\tan ^{2} \phi}\right)
$$

Rearranging the energy balance inequality, setting $B=2 d_{b}$, and substituting for $A_{\text {pers }}$,

$$
G_{e f f}>2 \gamma_{s}+2 \gamma_{s, g b} \frac{d_{b}}{2 n \delta_{g b}}\left(\frac{\tan \phi}{1+\tan ^{2} \phi}\right)
$$

The last term is the grain boundary resistance, $R_{g b}$. Currently, DISFRAC assumes the grain boundary surface energy is equal to the cleavage surface energy and there is only 1 breakthrough point, thus the grain boundary model in-use employs

$$
R_{g b}=2 \gamma_{s} \frac{d_{b}}{2 \delta_{g b}}\left(\frac{\tan \phi}{1+\tan ^{2} \phi}\right)
$$

In the code, the angular dependence is replaced by a more computationally efficient form: $\left(\frac{\tan \phi}{1+\tan ^{2} \phi}\right)=$ $0.5 \sin (2 \phi)$. The critical grain boundary separation, $\delta_{g b}$, is set to $18 \mathrm{~nm}$ based on the cohesive model employed by Kroon and Faleskog ${ }^{16}$. 


\section{DRAFT}

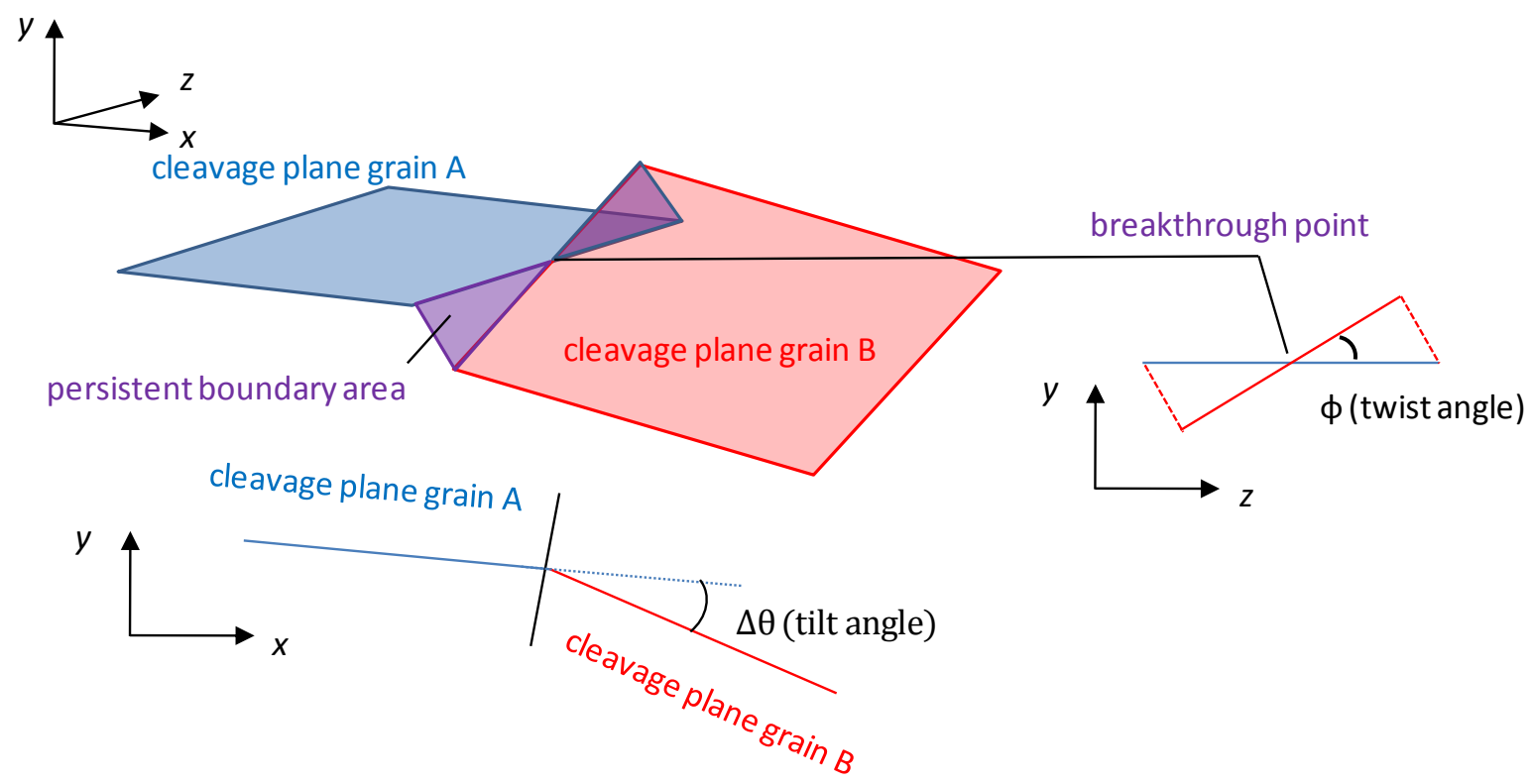

Figure 11. Misorientation of adjacent grains.

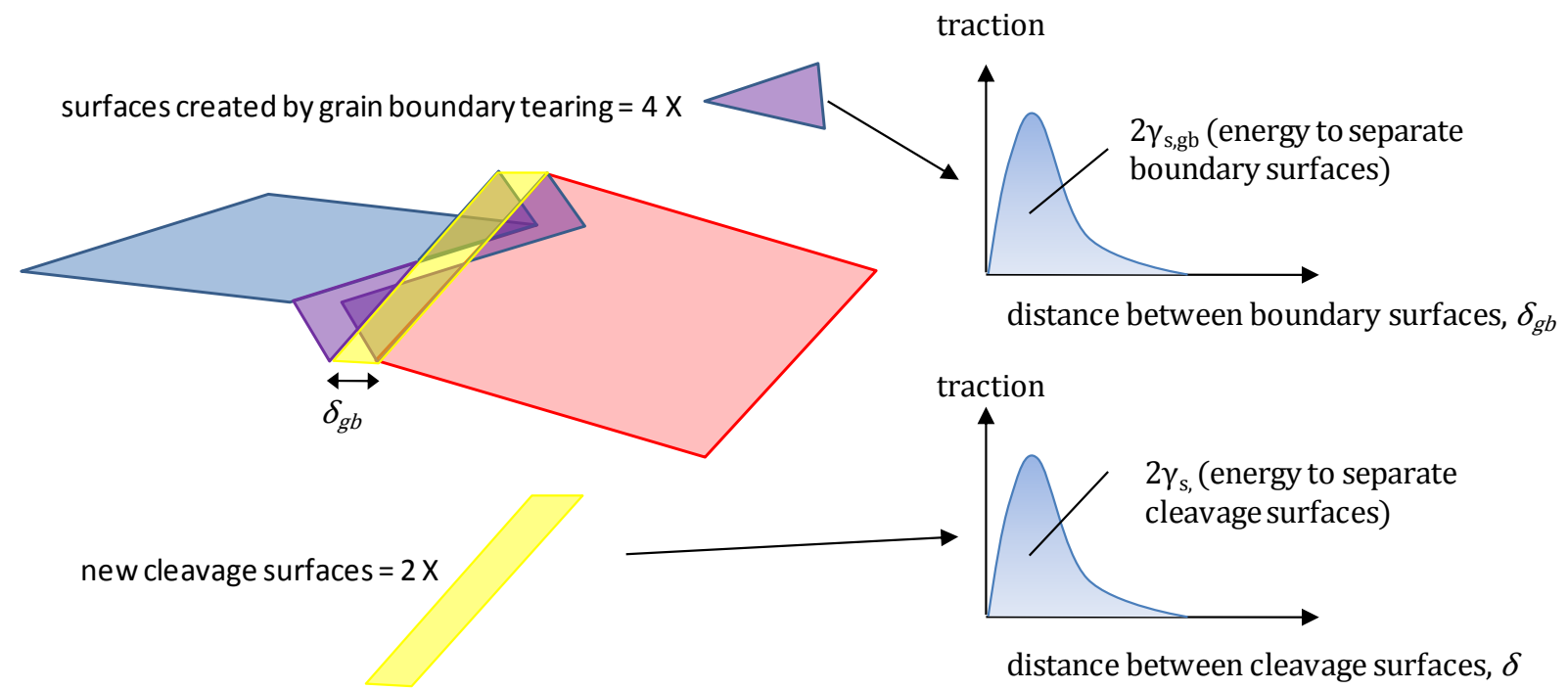

Figure 12. Grain boundary separation. Only a single breakthrough point shown. 


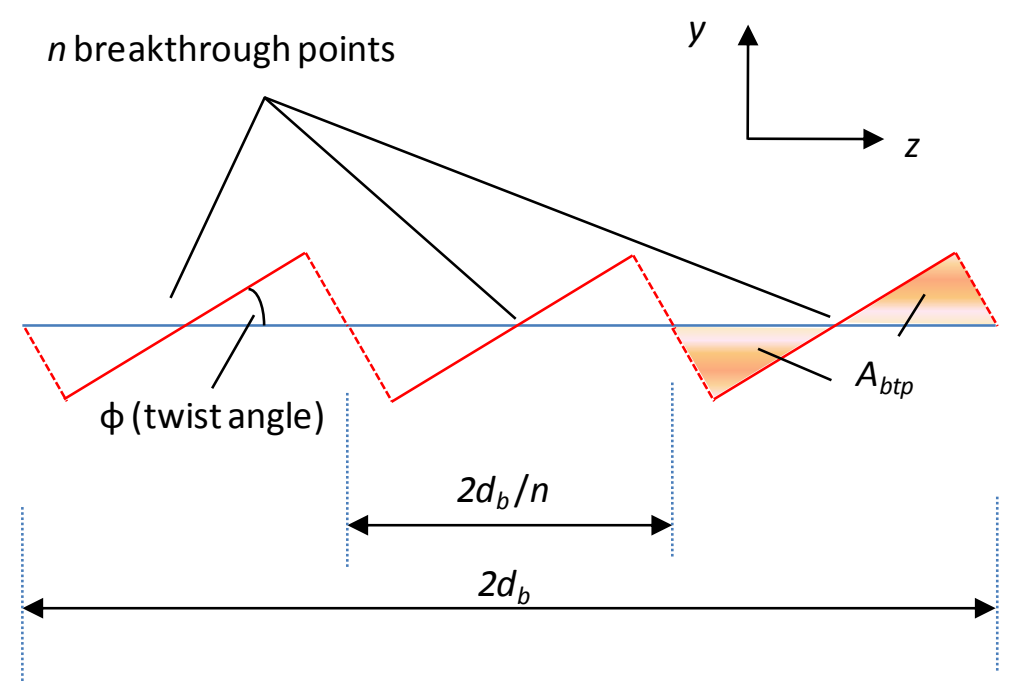

Figure 13. Persistent grain boundary area for multiple breakthrough points

\subsection{CRACK GROWTH IMPLEMENTATION}

For each particle, DISFRAC defines a potential path for cleavage crack growth through the surrounding grains. Figure 14 illustrates one such path. Each grain is defined by its size $(2 d)$ and orientation of its slip band $(\theta)$ with respect to the global $x$-axis. DISFRAC assumes that the active slip system lies within $\pm 45^{\circ}$ of the global $\mathrm{x}$-axis. For BCC crystals, the cleavage plane is $\beta=45^{\circ}$ from the slip plane. DISFRAC restricts the cleavage planes to within $\pm 45^{\circ}$ of the global $x$-axis; if the slip band angle is negative, the cleavage plane angle will be positive.

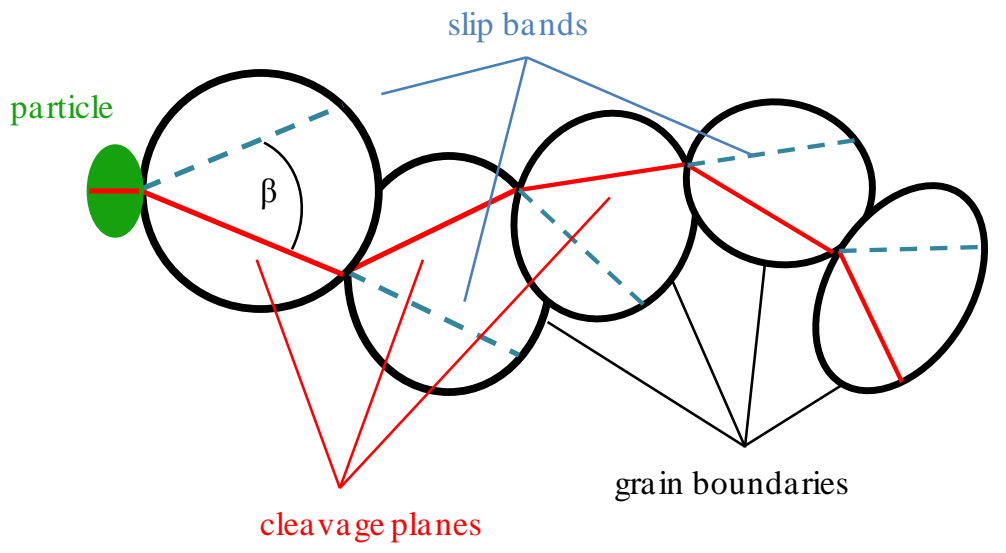

Figure 14. Crack path across grains

The actual crack path illustrated in Figure 14 is tortuous; defined by the red cleavage plane lines. DISFRAC, however, can accommodate only a straight microcrack in the Yokobori procedure for computing the stress-intensity of the microcrack and slip band. Thus, for each grain boundary energy evaluation, the microcrack length and orientation $(2 r$ and $\eta)$ correspond to an effective straight microcrack, starting at the center of the particle and terminating at the current grain boundary under evaluation. Figure 15 depicts the relevant geometric entities, for each phase of growth. 


\section{DRAFT}

In Figure 15a, the particle has cracked and DISFRAC must evaluate if the crack enters the adjacent grain. Here the microcrack is the full length of the particle and the cleavage plane and slip band in the first grain are randomly selected from the selected distributions. If the energy balance determines that the microcrack does cross this particle/grain boundary, the microcrack propagates across the first grain.

In Figure 15b, the effective microcrack, represented by the double black lines, is just slightly different than the actual crack path. Only half of the particle width is included; DISFRAC assumes (but does not follow) a separate microcrack propagating toward the macrocrack tip. The tilt misorientation $(\Delta \theta)$ uses the actual cleavage planes of the adjacent grains (both shown in red), not the effective microcrack direction.

Figure $15 \mathrm{c}$ and $\mathrm{d}$ demonstrate the sequence of microcrack growth, and each instance of the effective microcrack, if growth continues to be energetically favorable. The effective microcrack does underestimate the length of the actual microcrack, but should provide a reasonable approximation for most microcrack paths.

a)

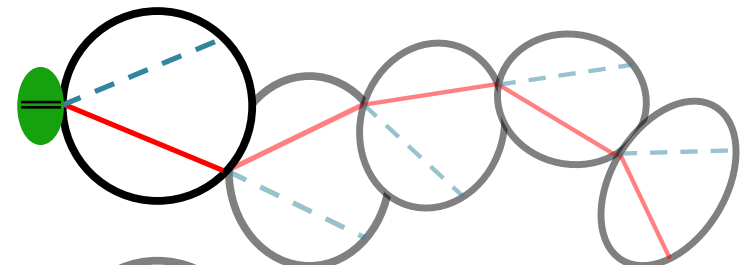

b)

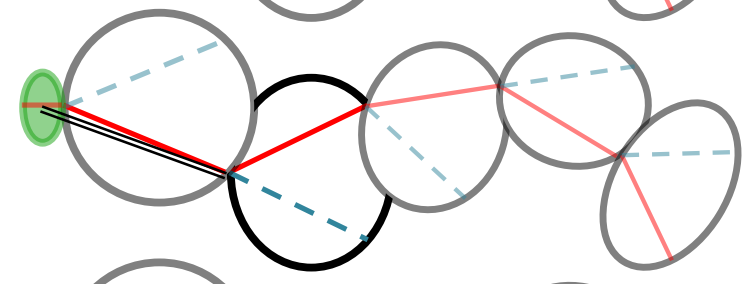

c)

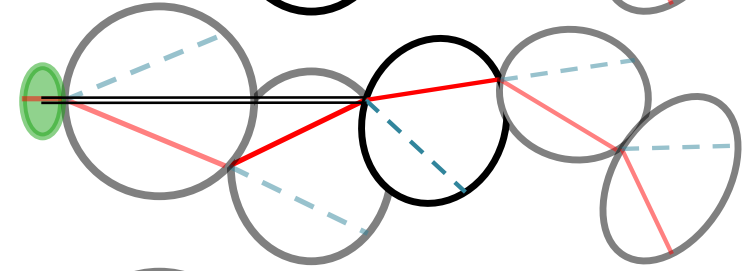

d)

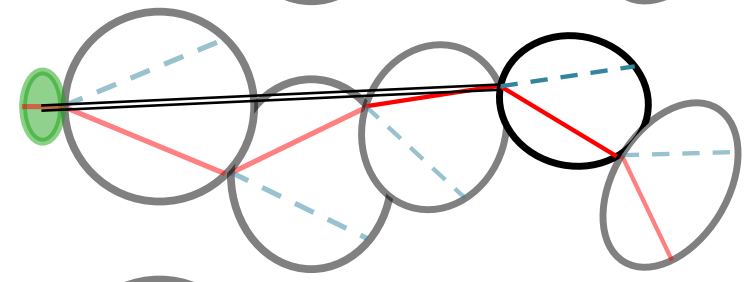

e)

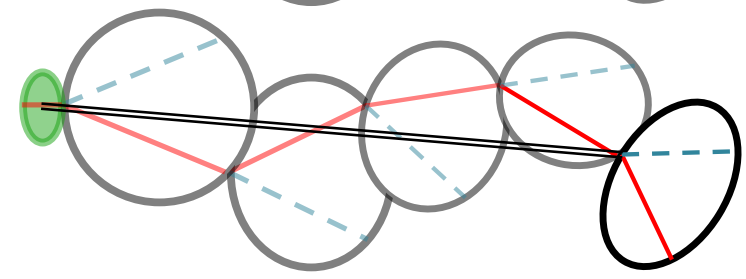

Figure 15. Growth stages of microcrack propagation 


\section{MACROCRACK STRESS FIELDS}

DISFRAC accounts for the elastic-plastic stress field ahead of the macrocrack tip with a database of finite element results from small-scale yielding, finite element-based, modified boundary layer analyses. Figure 16 presents the mesh employed in the nonlinear, finite-strain simulations with the commercial finite element software package ABAQUS.

At a given temperature, the stress profiles along a radial path from the macrocrack tip for various loads collapse into a single curve when normalized by the yield stress and J-integral representation of the load intensity. Thus, for each material (calibrated set of Zerilli-Armstrong model parameters) and constraint (T-stress) combination currently available, DISFRAC stores functional representations $\left(8^{\text {th }}\right.$ order Chebyshev polynomials) of these self-similar, normalized stress profiles at specific temperatures in the range of $-150^{\circ} \mathrm{C}$ to $+25^{\circ} \mathrm{C}$.

To access the global stress quantities at a point, DISFRAC computes the angular position from the macrocrack plane, $\omega$, and the normalized distance, $\bar{r}=r /\left(J / \sigma_{y}\right)$, from the stored polynomial representation at each temperature. Thus for each stress component $\left(\sigma_{x x}, \sigma_{y y}, \sigma_{x y}\right)$ and invariant $\left(\sigma_{\text {Mises }}, \sigma_{\text {mean }}\right)$, DISFRAC produces a table of values corresponding to each temperature case for the modified boundary layer (MBL) analyses. Piece-wise cubic Hermitian interpolation then produces the normalized stress values (e.g., $\sigma_{x x} / \sigma_{y}$ ) corresponding to the current DISFRAC analysis temperature. To use these stresses, DISFRAC multiplies by the yield stress and rotates to the correct reference axis if applicable.

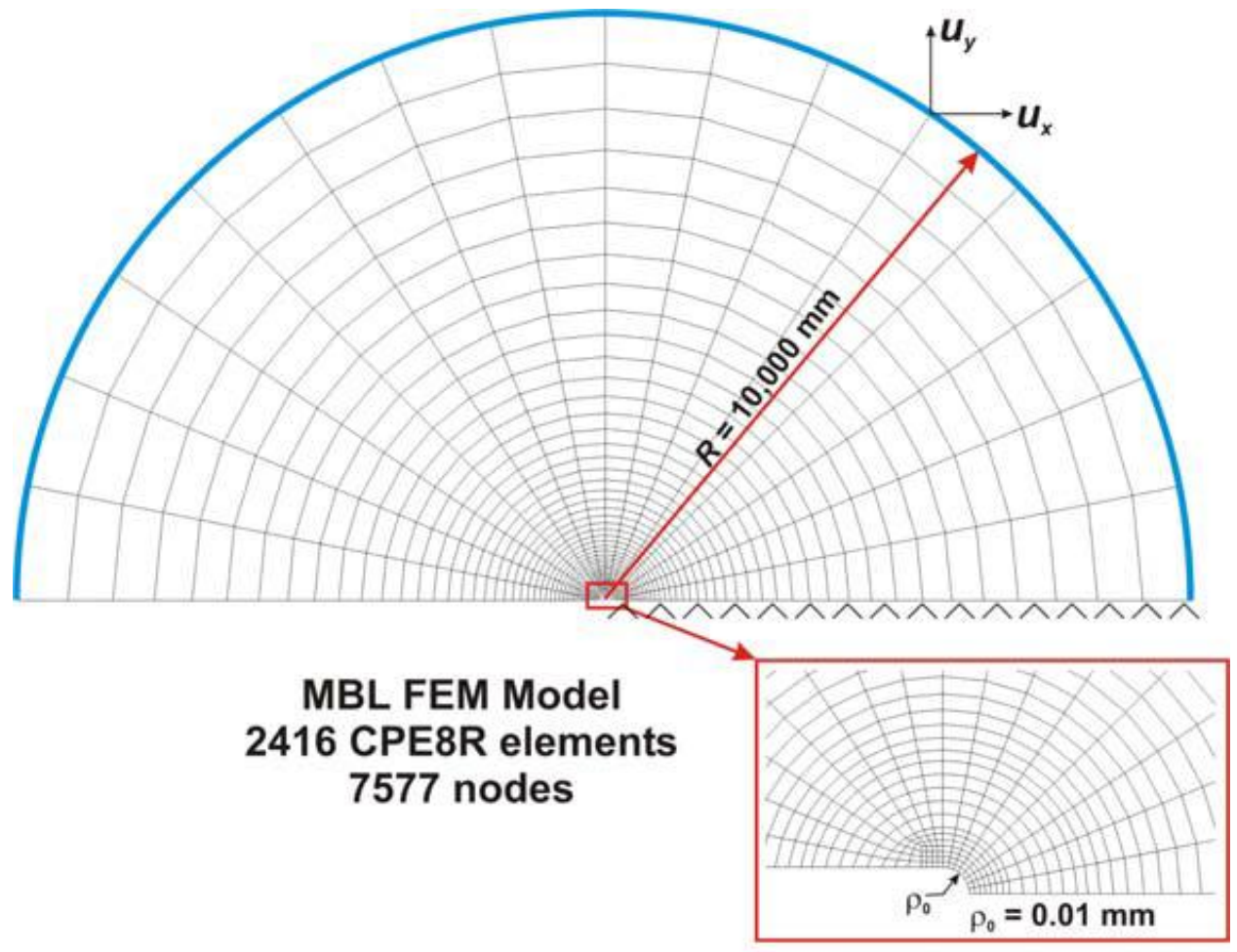

Figure 16. Modified boundary layer (MBL) finite element model (FEM) mesh 


\section{YOKOBORI DISLOCATION ANALYSIS}

Yokobori et al., ${ }^{9-12}$ developed a technique to compute stress intensity factors for a crack and slip band in interaction with each and an applied stress field. The crack consists of both a climb dislocation distribution, $f_{1}\left(x_{r}\right)$, and a glide dislocation distribution $f_{2}\left(x_{r}\right)$. A distribution of glide dislocations $f_{3}\left(x_{d}\right)$ comprises the slip band. Refer to Figure 17 for geometric definitions. The crack and slip band are separated by a distance $h$.

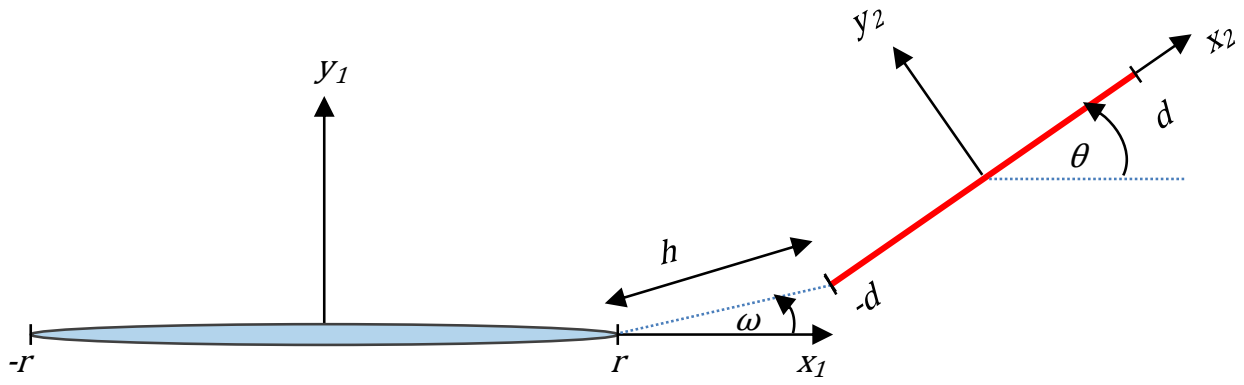

Figure 17. Yokobori analysis parameters

Enforcing static equilibrium between the linear-elastic stress fields of the dislocations and the applied stress field leads to the governing system of equations for the unknown dislocation distributions. Within the crack, the normal and shear stress must be zero and within the slip band the shear stress is zero. Thus, the total normal stress at a point $x_{r}$ within the crack is

$$
0=\sigma_{y, r}^{a p p}-A \int_{-r}^{r} \frac{f_{1}\left(\zeta_{r}\right)}{\zeta_{r}-x_{r}} d \zeta_{r}+A\left[\int_{-d}^{d}\left(K_{31}\left(x_{r}, \zeta_{d}\right) * f_{3}\left(\zeta_{d}\right)\right) d \zeta_{d}\right]
$$

where $A=G b / 2 \pi(1-v), \sigma_{y y 1}^{a p p}$ is the normal component (relative to the crack plane) of the applied stress field, $\zeta_{d}$ is a point within the slip band and $K_{31}$ is an interaction term describing the normal stress at a point in the crack due to a glide dislocation within the slip band. Similarly, summing the shear stress at points within the crack and slip band provide the following two equations.

$$
\begin{gathered}
0=\tau_{r}^{a p p}-A \int_{-r}^{r} \frac{f_{2}\left(\zeta_{r}\right)}{\zeta_{r}-x_{r}} d \zeta_{r}+A\left[\int_{-d}^{d}\left(K_{32}\left(x_{r}, \zeta_{d}\right) * f_{3}\left(\zeta_{d}\right)\right) d \zeta_{d}\right] \\
0=\tau_{d}^{e f f}-A \int_{-d}^{d} \frac{f_{3}\left(\zeta_{d}\right)}{\zeta_{d}-x_{d}} d \zeta_{d} \\
+A\left[\int_{-r}^{r}\left(\left(K_{13}\left(x_{d}, \zeta_{r}\right) * f_{1}\left(\zeta_{r}\right)\right)+\left(K_{23}\left(x_{d}, \zeta_{r}\right) * f_{2}\left(\zeta_{r}\right)\right)\right) d \zeta_{r}\right]
\end{gathered}
$$




\section{DRAFT}

Here, $\tau_{r}^{a p p}$ is the shear component of the applied stress field relative to the crack plane. The effective shear stress on the slip band, $\tau_{d}^{\text {eff }}$, is $\operatorname{sgn}\left(\tau_{d}^{a p p}\right)\left[\max \left(\left|\tau_{d}^{a p p}\right|-\tau_{f}, 0\right)\right]$, where $\tau_{d}^{a p p}$ is the shear component of the applied stress field relative to the slip band and $\tau_{f}$ is the friction stress.

The interaction terms are

$$
\begin{gathered}
K_{31}=\frac{-y_{d r}\left(x_{d r}^{2}+y_{d r}^{2}\right)+2 y_{d r} x_{d r}^{2} \cos (2 \theta)+x_{d r}\left(x_{d r}^{2}-y_{d r}^{2}\right) \sin (2 \theta)}{\left(x_{d r}^{2}+y_{d r}^{2}\right)^{2}} \\
K_{32}=\frac{-2 y_{d r} x_{d r}^{2} \sin (2 \theta)+x_{d r}\left(x_{d r}^{2}-y_{d r}^{2}\right) \cos (2 \theta)}{\left(x_{d r}^{2}+y_{d r}^{2}\right)^{2}} \\
K_{13}=\frac{2 x_{r d} y_{r d}^{2} \sin (2 \theta)+y_{r d}\left(x_{r d}^{2}-y_{r d}^{2}\right) \cos (2 \theta)}{\left(x_{r d}^{2}+y_{r d}^{2}\right)^{2}} \\
K_{23}=\frac{2 y_{r d} x_{r d}^{2} \sin (2 \theta)+x_{r d}\left(x_{r d}^{2}-y_{r d}^{2}\right) \cos (2 \theta)}{\left(x_{r d}^{2}+y_{r d}^{2}\right)^{2}}
\end{gathered}
$$

The subscripts on the distance variables correspond to their definition; e.g., $x_{d r}$ is the distance from point $\zeta_{d}$ to $x_{r}$ along the axis of the slip band. Thus,

$$
\begin{gathered}
x_{d r}=-\left(c-x_{r}\right) \cos (\theta)-h \cos (\theta-\omega)-\left(d+\zeta_{d}\right) \\
y_{d r}=\left(c-x_{r}\right) \sin (\theta)+h \sin (\theta-\omega) \\
x_{r d}=\left(c-\zeta_{r}\right) \cos (\theta)+h \cos (\omega)+\left(d+x_{d}\right) \cos (\theta) \\
y_{r d}=h \sin (\omega)+\left(d+x_{d}\right) \sin (\theta)
\end{gathered}
$$

The solution procedure includes the following assumptions.

- The distributions are infinite at the tips of the crack and slip band; i.e., $f_{1}( \pm r)=f_{2}( \pm r)=$ $f_{3}( \pm d)= \pm \infty$. Thus the distributions are given the form $f(x)=h(x) / \sqrt{a^{2}-x^{2}}$ where a is $1 / 2$ the length of the distribution ( $d$ or $r$ ) and $h(x)$ is a non-singular function. The nonsingular functions inform the stress intensity factors.

$$
\begin{aligned}
& f_{1}\left(x_{r}\right)=h_{1}\left(x_{r}\right) / \sqrt{r^{2}-x_{r}^{2}} \\
& f_{2}\left(x_{r}\right)=h_{2}\left(x_{r}\right) / \sqrt{r^{2}-x_{r}^{2}} \\
& f_{3}\left(x_{d}\right)=h_{1}\left(x_{d}\right) / \sqrt{d^{2}-x_{d}^{2}}
\end{aligned}
$$


- The numbers of positive and negative dislocations are equal within each distribution. This is equivalent to enforcing that the relative displacements of the crack surfaces are zero at the tip.

The governing equations are inverted following the techniques of Muskhelishivili yielding a system of simultaneous integral equations. These are then solved by discretizing the crack and slip band and forming an algebraic system of equations for the value of the unknown $h(x)$ functions at each point.

The stress intensity factors at the right tip of the crack $\left(K_{I r}, K_{I I r}\right)$ and the left tip of the slip band $\left(K_{I I d}\right)$ depend only of the value of the corresponding $h$ functions and material and geometric constants.

$$
\begin{gathered}
K_{I r}=\pi A \sqrt{\frac{\pi}{r}} h_{1}(r) \\
K_{I I r}=\pi A \sqrt{\frac{\pi}{r}} h_{2}(r) \\
K_{I I d}=-\pi A \sqrt{\frac{\pi}{d}} h_{3}(-d)
\end{gathered}
$$

Within DISFRAC, the right-hand microcrack and left-hand slip band coincide; i.e., $h=0$. (Note this is $h$ for the Yokobori solution not $h$ for the general DISFRAC setup which sets the distance from the macrocrack to the particle). The Yokobori equations as described above, and in the original works, assume the microcrack lies along the global $\mathrm{x}$-axis. This is not generally the case within DISFRAC. Nevertheless, the equations may be used without modification with proper selection of variables. In the DISFRAC code, the Yokobori variable $\theta$ describes the angle between the microcrack and slip band (i.e. $\theta-\eta$ for the DISFRAC variables, see Figure 1). The loading terms, $\sigma_{y, r}^{a p p}, \tau_{r}^{a p p}$ and $\tau_{d}^{a p p}$ come from the macrocrack-induced stress field (via the FEM solutions), rotated with respect to either the microcrack or slip band axis as necessary. 


\section{MATERIAL BEHAVIOR}

\subsection{ZERILLI-ARMSTRONG MATERIAL MODEL}

\subsubsection{Description}

The continuum constitutive model developed by Zerilli and Armstrong ${ }^{17}$ governs the macroscopic stressstrain response, the yield stress temperature dependence, the flow stress temperature dependence, and the relationship between the von Mises stress and the equivalent plastic strain. The so-called ZerilliArmstrong equation derives its temperature dependence from the activation energy of a material's short range obstacles. Obstacles to dislocation motion are microstructural features of the material (precipitates, solutes, grain boundaries, etc.) that provide a larger barrier to dislocation motion than do the lattice atoms. Thus, the dislocations must have a larger driving force to pass these features either by cutting them, or climbing around them, or, as in the case of interstitials and vacancies, simply moving through their increased strain field. These barriers are divided into short and long-range barriers according to whether an input of thermal energy into the material provides a significant reduction in the activation energy required by the dislocation to move around them or not. Zerilli and Armstrong determined that the only short-range obstacle in BCC metals is the lattice atoms themselves and so the temperature dependence of the flow behavior for BCC metals is a function of the lattice spacing. Therefore ferritic steels, which all have the same lattice structure as $\alpha$-iron and consequently the same lattice spacing, should all exhibit the same temperature dependent flow behavior. The equation formulated by Zerilli and Armstrong is shown below in a slightly updated form to describe the temperature dependence of BCC metals.

$$
\sigma_{Z A}=\sigma_{G}+\frac{k_{y}}{\sqrt{2 d}}+\mathrm{K} \sqrt{\epsilon}+B e^{-\left(\beta_{0}-\beta_{1} \ln \dot{\epsilon}\right) T}
$$

If the material exhibits dynamic strain aging (DSA), then the material constant, $K$, describing strain hardening, becomes a temperature dependent parameter:

$$
\mathrm{K}=B_{0} e^{-\left(\alpha_{0}-\alpha_{1} \ln \dot{\epsilon}\right) T}
$$

The first two terms of Eqn. 49 represents the yield strength while the third term is the work hardening behavior. The last term represents the temperature dependence of moving the dislocations past the lattice atoms. Temperature is represented by $T, \epsilon$ is the strain and $\dot{\epsilon}$ is the strain rate. All other constants are material specific parameters. The yield strength of BCC materials is very sensitive to temperature and strain rate while the work hardening behavior is not (except under conditions of DSA) as microstructural features other than the lattice atoms (including other dislocations, precipitates, vacancy and interstitial clusters, etc) are all considered to be long range obstacles to dislocation motion, not affected by temperature. The effects of these features on the movement of dislocations are included in the athermal terms in Eqn. 49. 


\subsubsection{Usage}

\subsubsection{Equivalent plastic strain}

In DISFRAC, the plastic strain depends on the current Mises equivalent stress via the Zerilli-Armstrong relationship as

$$
\epsilon_{p l}=\left[\frac{\sigma_{\text {Mises }}-\sigma_{G}-k_{y} / \sqrt{2 d}-B e^{-\left(\beta_{0}-\beta_{1} \ln \dot{\epsilon}\right) T}}{B_{0} e^{-\left(\alpha_{0}-\alpha_{1} \ln \dot{\epsilon}\right) T}}\right]^{2}
$$

DISFRAC imposes a maximum plastic strain of 20.0. Note that average grain size is used for $d$, not the actual size of a specific grain.

\subsubsection{Yield stress}

In DISFRAC, the yield stress is the Zerilli-Armstrong equivalent stress for $\epsilon_{p l}=0$. Note that average grain size is used for $d$, not the actual size of a specific grain.

$$
\sigma_{y}=\sigma_{G}+k_{y} / \sqrt{2 d}+B e^{-\left(\beta_{0}-\beta_{1} \ln \dot{\epsilon}\right) T}
$$

\subsubsection{Elastic friction stress}

DISFRAC uses the elastic friction stress - the friction stress at zero plastic strain - to govern dislocation motion..

$$
\tau_{f}=\frac{1}{\sqrt{3}}\left[\sigma_{G}+B e^{-\left(\beta_{0}-\beta_{1} \ln \dot{\epsilon}\right) T}\right]
$$

\subsubsection{Finite element analysis}

For each material parameter set available, a series of finite element analyses employed the ZerilliArmstrong constitutive model via user-defined strain hardening curves within ABAQUS. For each temperature and strain rate, computed pairs of stress and plastic strain values from the Zerilli-Armstrong equation were supplied as input to the finite element program. Note that the finite element analyses are quasi-static and the strain rate remains constant.

\subsection{ELASTIC PROPERTIES}

DISFRAC computes the temperature dependent shear and elastic moduli with the following equations. 


\section{DRAFT}

Poisson's ratio is a user input. $T_{K}$ indicates the current analysis temperature in Kelvin.

$$
\begin{array}{cr}
G=81.0 E 3 *\left[1-\frac{1.09\left(T_{K}-300\right)}{1810}\right] ; & T_{K} \leq 573 \mathrm{~K} \\
G=81.0 E 3 *\left[1-\frac{1.09\left(T_{K}-300\right)}{1810}\right]-0.032\left(T_{K}-573\right)^{2} ; & 573 K<T_{K} \leq 923 \mathrm{~K} \\
G=81.0 E 3 *\left[1-\frac{1.09\left(T_{K}-300\right)}{1810}\right]-0.032\left(T_{K}-573\right)^{2}-0.024\left(T_{K}-923\right)^{2} ; T_{K}>923 \mathrm{~K} \\
E=2 G(1+v)
\end{array}
$$




\section{REFERENCES}

1. Regulatory Guide 1.154, "An Approach for Using Probabilistic Risk Assessment in RiskInformed Decisions on Plant-Specific Changes to the Licensing Basis," U.S. Nuclear Regulatory Commission, January 1987.

2. ASTM E1921-97, "Standard Test Method for Determination of Reference Temperature, To, for Ferritic Steels in the Transition Range," ASTM Vol. 3.01, West Conshohoken PA, USA: American Society for Testing and Materials, 1999.

3. Williams, T., D.I. Swan, and G.L. Dixon, "Modification of the Lower Tail of the Master Curve Distribution," Embrittlement and Mechanistic Interpretation of Reactor Pressure Vessel and Internal Materials, European Commission report, EUR 12835 EN, ISBN 92-7900173-6 European Committee, 2005

4. Wallin , K., R. Rintamaa, "Statistical Re-Evaluation of the ASME KIC and KIR Fracture Toughness Reference Curves," VTT Manufacturing Technology, Finland, 23rd MPASeminar, Stuttgart, October 1 and 2, 1997

5. EricksonKirk, M.T., "Probabilistic Fracture Mechanics: Models, Parameters, and Uncertainty Treatment Used in FAVOR Version 04.1," USNRC report NUREG-1807, Dec. 2006

6. Marston, T. U., August 1978, "Flaw Evaluation Procedures: Background and Application of ASME Section XI Appendix A,” Electric Power Research Institute Report No. NP-719SR.

7. Xia, L. and Cheng, L., Transition from ductile tearing to cleavage fracture: A cell-model approach, Int. J. Fracture, 1997, 87, 289-306.

8. Chiou Jr., W. C. and Carter, E., 2003, "Structure and stability of $\mathrm{Fe}_{3} \mathrm{C}$-cementite surfaces from first principles," Surf. Sci., 530, pp. 87-100.

9. Yokobori, T., 1968, "Criteria for Nearly Brittle Fracture,” Int. J. Frac. Mech., 4, pp. 179-204.

10. Yokobori, T. and Kamei, A., 1973, "A Criterion for Low-Stress Brittle Fracture of Materials with Crack or Sharp Notch Based on Combined Micro-and-Macro Fracture Mechanics," Eng. Fract. Mech., 5, pp. 819-836.

11. Yokobori, T., Kamei, A., and Konosu, S., 1976, "A Criterion for Low-Stress Brittle Fracture of Notched Specimens Based on Combined Micro- and Macro Fracture Mechanics - I," Eng. Fract. Mech,. 8, pp. 397-409.

12. Yokobori, T., Sawaki, Y., and Nakanishi, S., 1979, "Criterion for Brittle Fracture of Notched or Cracked Specimens Based on Combined Micro- and Macro Crack Mechanics II,” Eng. Fract. Mech., 12, pp. 125-141.

13. Weertman J. 1996, Dislocation Based Fracture Mechanics. World Scientific, Singapore.

14. Wallin, K., and Laukkanen, A., 2006, "Aspects of cleavage fracture initiation - relative influence of stress and strain," Fatigue Fract. Engng Mater Struct, 29, pp. 788-798.

15. Wallin, K., and Laukkanen, A., 2008, "New Developments of the Wallin, Saario, Torronen cleavage fracture model," Eng. Fract. Mech, 75, pp. 3367-3377.

16. M. Kroon and J. Faleskog, 2005, "Micromechanics of cleavage fracture initiation in ferritic steels by carbide cracking”, J. Mech Phys. Solids, 53, pp. 171-196.

17. Zerilli, F. J., and Armstrong R. W., 1967, "Dislocation-Mechanics-Based Constitutive Relations for Material Dynamics Calculations," J App Phys, 61(5), pp. 1816-1825.

18. Anderson, T. L., 1994, Fracture Mechanics: Fundamentals and Applications, CRC Press, Boca Raton.

19. Y. Qiao and A.S.Argon, "Cleavage crack-growth-resistance of grain boundaries in polycrystalline $\mathrm{Fe}-2 \% \mathrm{Si}$ alloy: experiments and modeling," Mechanics of Materials 35 (2003) 129-154 


\section{DRAFT}

20. Y. Qiao and A.S.Argon, "Cleavage cracking resistance of high angle grain boundaries in Fe-3\%Si alloy," Mechanics of Materials 35 (2003) 313-331

21. Y. Qiao and X. Kong, "An energy analysis of the grain boundary behavior in cleavage cracking in Fe-3wt.\%Si alloy," Materials Letters 58 (2004) 3156-3160

22. Cochran, K.B. et al, "A dislocation-based cleavage initiation model for pressure vessel steels” PVP2012-78564. July 15-19, 2012 Toronto, Ontario, Canada 


\section{APPENDIX A. LINEAR ELASTIC FRACTURE MECHANICS (LEFM) BACKGROUND INFORMATION}

As a crack grows, the potential energy of a specimen decreases. The energy release rate, $G$, is defined as the negative of the change in potential energy (making $G$ a positive quantity)

$$
G=-\frac{d \Pi}{d A}
$$

Thus, $G$, is the energy available to grow the crack by $d A$. In linear elastic materials, $G$ is proportional to $K^{2}$, where $K$ is the stress intensity factor. The stress intensity factors set the magnitudes of stress and displacement near the linear-elastic crack tip. The spatial variations of the stress and displacement fields depend on the loading mode (opening, in-plane shear, out-of-plane shear). For all 3 modes, the non-zero stress components exhibit $1 / \sqrt{r}$ singularity with distance $(r)$ from the crack tip while the non-zero displacements vary with $\sqrt{r}$.

A method to compute the energy release rate considers the work required to close a length $(d a)$ of an open crack tip. This involves the product of stress and displacement which is finite (and independent of $r$ ). The result depends only on the stress intensity factors and material properties, see Anderson ${ }^{18}$ for complete derivation. In mixed mode (I and II) loading (assuming no change of crack shape or direction)

$$
G=\frac{K_{I}^{2}}{E^{\prime}}+\frac{K_{I I}^{2}}{E^{\prime}}
$$

with $E^{\prime}=E$ in plane stress and $E^{\prime}=E /\left(1-v^{2}\right)$ in plane strain.

At a grain boundary, the crack changes directions as it transitions to the cleavage plane of the next grain. Following Anderson's treatment of an infinitesimal kink, the energy release rate depends on stress intensity factors local to the new crack direction. The angle $\alpha$ indicates the angle from the existing crack plane to the plane of the new crack direction, counter clockwise positive.

$$
G(\alpha)=\frac{k_{I}^{2}(\alpha)+k_{I I}^{2}(\alpha)}{E^{\prime}}
$$

Here, $k_{I}(\alpha)$ and $k_{I I}(\alpha)$ are the stress intensity factors at the tip of the kink, which depend on the stress-intensity factors for the main crack $\left(K_{I}, K_{I I}\right)$ and geometric coefficients:

$$
\begin{aligned}
& k_{I}(\alpha)=\sigma_{y y} \sqrt{2 \pi r}=C_{11} K_{I}+C_{12} K_{I I} \\
& k_{I I}(\alpha)=\tau_{x y} \sqrt{2 \pi r}=C_{21} K_{I}+C_{22} K_{I I}
\end{aligned}
$$




\section{DRAFT}

$$
\begin{aligned}
& C_{11}=\frac{3}{4} \cos \left(\frac{\alpha}{2}\right)+\frac{1}{4} \cos \left(\frac{3 \alpha}{2}\right) \\
& C_{12}=-\frac{3}{4}\left[\sin \left(\frac{\alpha}{2}\right)+\sin \left(\frac{3 \alpha}{2}\right)\right] \\
& C_{21}=\frac{1}{4}\left[\sin \left(\frac{\alpha}{2}\right)+\sin \left(\frac{3 \alpha}{2}\right)\right] \\
& C_{22}=\frac{1}{4} \cos \left(\frac{\alpha}{2}\right)+\frac{3}{4} \cos \left(\frac{3 \alpha}{2}\right)
\end{aligned}
$$

These factors apply to both the left and right tips of the crack provided $\alpha$ is taken to be positive counter-clockwise, see figure below. The coefficients control both the magnitude and sign of the resultant stress-intensity factors. For example, positive mode I opening of the existing crack contributes only to positive mode I loading in the new direction $\left(C_{11}>0\right.$ for all $\left.\alpha\right)$ while positive mode I crack opening produces positive shear opening for $\alpha>0$ but negative shear opening for $\alpha<0$ (via $C_{21}$ ). Note also that a positive main crack shear opening leads to negative mode I opening for $\alpha>0$ (via $C_{12}$ ); effectively putting the new crack direction in compression.

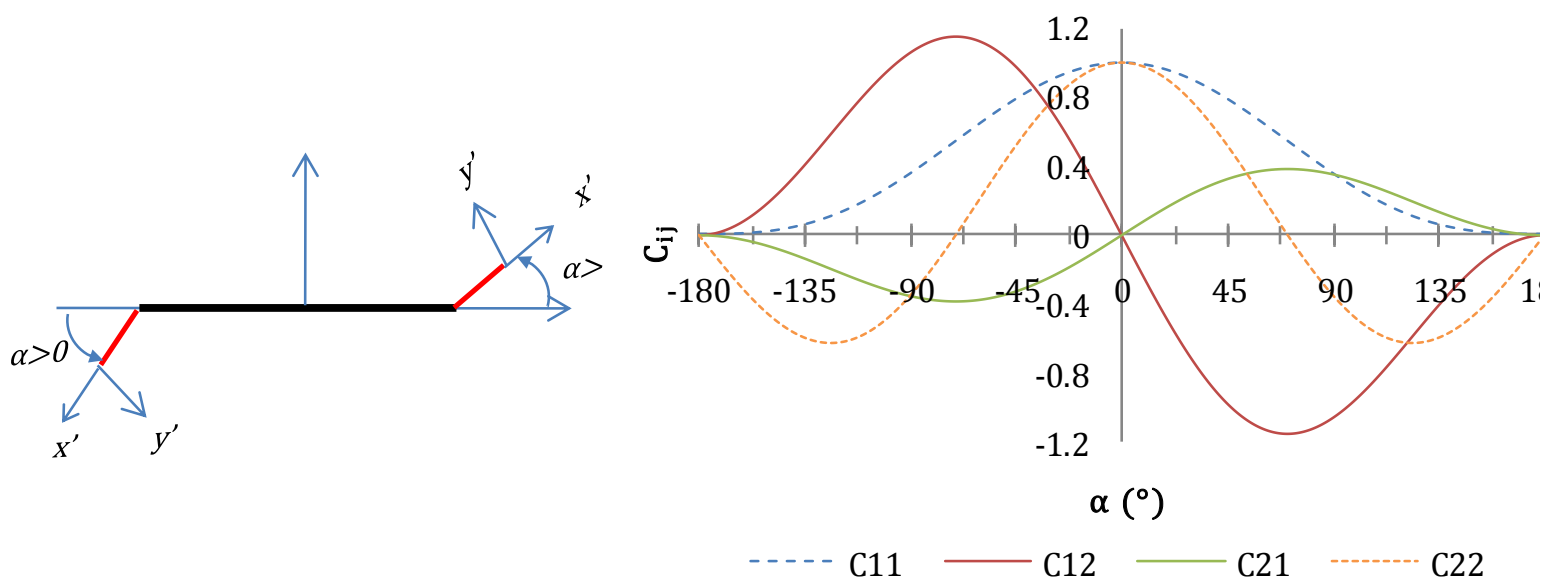

Figure 18. Angle definitions and coefficient dependence on $\alpha$ for stress-intensity factors of crack with infinitesimal direction change. 